\title{
Praktisches Handbuch der
}

Fre i ma rk e n des

Königreichs Sachsen und des Herzogtums Sachsen-Altenburg mit Beschreibung und Bewertung der

\section{Abstempelungen}

sowie Orts-und Schlagwortregister G3(2) 155

$19 ? 21$

NPM

Jnter Mitarbeit von Walter A. O pitz, Netzschkau i. V. herausgegeben von

Friedrich W. Dieck

Leipzig

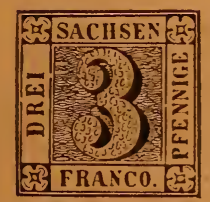

Selbstverlag des Verfassers Fr. W. Dieck, Leipzig, Nordplatz 5 1921

Auslieferung durch die Kommissionsbuchhandlung L. Fernau, Leipzig, Talstr. 15 



\section{Praktisches Handbuch der}

Freimarken

\section{des \\ Königreichs Sachsen}

und des Herzogtums Sachsen-Altenburg

mit Beschreibung und Bewertung der

\section{Abstempelungen}

sowie Orts - und Schlagwortregister

Unter Mitarbeit von Walter A. Opitz, Netzschkau i. Vgtl.

herausgegeben von

Friedrich W. Dieck

Leipzig

Selbstverlag des Verfassers Fr. W. Dieck, Leipzig, Nordplatz 5 1921 
Alle Rechte, besonders auch das Recht der Übersetzung, vorbehalten.

Abdruck einzelner Abschnitte nur mit genauer Quellenangabe und mit Einverständnis des Herausgebers gestattet. 


\section{Vorwort.}

Da sämtliche, die Sachsenmarken behandelnden Schriften schon seit Jahren im Buchhandel nicht mehr zu bekommen und die in den Büchereien vorhandenen Werke meist stark veraltet sind, ist der Plan zur Herausgabe dieses kurzen Handbuches entstanden. Es soll nicht nur, unter Fortlassung vieler für den praktischen Gebrauch nicht unbedingt notwendiger Einzelheiten, die hauptsächlichsten geschichtlichen Daten kurz zusammenfassen, sondern auch das schwierige Gebiet der Abstempelungen ausführlicher behandeln.

Die erstmalig aufgestellten Preistafeln sind als ungefähre Grundlage für den Erwerb und Tausch der verschiedenen Abstempelungen gedacht.

Zu besonderem Dank bin ich Herrn Walter A. Opitz, Netzschkau i. Vogtl., verpflichtet, der seine reichen Erfahrungen besonders auf dem Gebiete der Abstempelungen zur Verfügung gestellt hat. Die Herren Max Thier, Charlottenburg, A. Dietrich und E. Ehrlich, Halle a. S., Oberlehrer Große in Grimma, E. Mancke, E. Marré und Oberlehrer Voigt in Leipzig haben mich in dankenswerter Weise mit Ratschlägen, Verbesserungen und Ergänzungen unterstützt, während Herr Faktor Seifert vom Vorstand des Germania-Ringes die Drucklegung in promptester Weise besorgt hat.

Auch an meine Leser möchte ich die Bitte richten, ihnen auffallende Fehler zu berichtigen und Vorschläge über wünschenswerte Zusätze zu machen. Hauptsächlich auf dem Gebiete der Abstempelungen und unter diesen besonders bei den Briefsammlungen dürften sich durch weitere Forschungen noch Ergänzungen möglich machen.

Leipzig, Nordplatz 5, Fernsprecher 6311 im Oktober 1921.

Fr. W. Dieck. 



\section{I.}

Schon seit Ende des 15. Jahrhunderts bestanden in verschiedenen deutschen Landen sogenannte Boten- und Hofposten, wie z. B. in Dresden die Hofpost des Kurfürsten August von Sachsen, jedoch erfolgte die Entwicklung der "Post" zur öffentlichen Verkehrseinrichtung erst im Laufe des 16. Jahrhunderts.

Der deutsche Kaiser Rudolph II. ernannte 1595 Leonhard von Taxis zum Reichsfreiherrn und General-Oberpostmeister über die Posten im Heiligen römischen Reich deutscher Nation. Kaiser Matthias belehnte dann 1615 Lamoral von Taxis erblich mit dem Reichspostmeisteramt.

Eine Anzahl deutscher Fürsten, darunter auch der Kurfürst August von Sachsen, verzichteten jedoch nicht auf ihr Postrecht, sondern legten, teilweise sogar neben den Taxisschen und anderen Postlinien, eigene öffentliche Posten an. So war zum Beispiel der Leipziger Posthalter Johann Sieber im Anfange des 17. Jahrhunderts gleichzeitig Rats-Botenmeister, Kurfürstlich Sächsischer Postmeister und Kaiserlicher Postmeister für die Botenpost nach Frankfurt a. M.

Im Jahre 1721 schloB das benachbarte Sachsen-Altenburg für 60 Taler jährliche Pacht eine Postkonvention mit Kursachsen $a b$, übernahm jedoch 1782 das Postwesen in eigene Verwaltung, übertrug 1818 die Besorgung der Post der Fürstlich Thurn- und Taxisschen Postverwaltung, um 1822 teilweise und nach Eröffnung der Eisenbahn Leipzig-Altenburg am 1. August 1847 sein gesamtes Postwesen 
wiederum auf 25 Jahre gegen 600 Taler jährliche Pacht an die Sächsische Regierung zu verpachten. Am 15. Mai 1850 trat Sachsen mit Sachsen-Altenburg dem Deutsch-Österreichischen Postverein bei, dem außer Preußen und Österreich damals noch Bayern, Mecklenburg-Strelitz und Schleswig-Holstein angehörten. Beide Postgebiete gingen dann am 1. Januar 1868 im Norddeutschen Postbezirk auf und gebrauchten vom 1. Januar 1872 ab die deutschen Reichspostmarken in der Groschenwährung. 
1. Buchdruckausgabe vom 29. Juni 1850 , im Gebrauch ab 1. Juli 1850 .

Durch seinen Beitritt zum Deutsch-Österreichischen Postverein hatte Sachsen am 15. Mai 1850 die Verpflichtung übernommen, „Francozeichen" einzuführen, wie es ja schon seit 1653 Frankostreifbänder der Pariser Stadtpost und seit dem 6. Mai 1840 die bekannte schwarze englische Pennymarke gab. Während nun letztere Marke als Vorbild für die erste schwarze Bayernmarke gedient hat, bildeten wiederum die ersten Ausgaben der bayrischen Kreuzermarken das Vorbild für die erste sächsische Marke, die heute weltberühmte

\section{sächsische Zeitungsinarke 3 Pfennige rot,}

die in verschiedenen Farbarten von hellziegelrot bis karmin und kirschrot vorkommt.

Der mit der Bestellung der Marke beauftragte Leipziger Oberpostrat von Schimpff ließ von dem Litographen und Buchdruckereibesitzer J. B. Hirschfeld in Leipzig, Neumarkt 29, dessen Druckerei noch heute von dem jetzigen Besitzer Arno Pries in der Brüderstraße weitergeführt wird, nach dem Muster der bayrischen Marken Zeichnungen und schwarze Probedrucke anfertigen. Nach Bestätigung der endgültigen Zeichnung und Wahl der roten Farbe wurden dann im Juni 1850 die ersten 
120000 Stück in rotem Druck auf weißem Papier in Auftrag gegeben und von Hirschfeld noch in demselben Monat zu je 20 Stück auf einem Bogen gedruckt.

Die Originalzeichnung wurde in Holz geschnitten, der Druck selbst erfolgte von den in der Hirschfeldschen Stereotypie hergestellten Bleiabgüssen, welche in 4 Reihen zu je 5 Stück mit Satzlinien zwischen den einzelnen Marken zu einem Bogen zusammengesetzt wurden. Der Druck der ersten Auflage wurde von dem Druckereifaktor Meyn auf halben Oktavbriefbogen auf der Handpresse ausgeführt. Im ganzen sind von Hirschfeld vom Juni 1850 bis 17. Juni 1851 in 8 Auflagen 25000 Bogen oder

500000 Stück rote Dreiermarken

gedruckt und abgeliefert worden.

Von dieser Menge ist 1 Bogen oder 20 Stück als Beleg an das Finanzministerium nach Dresden gegangen. Der Bogen ist dann später zerschnitten worden und die Marken wurden zu M. 3.- für das Stück an Sammler mit ,guten Verbindungen" abgegeben.

463058 Stück sind bis zum 12. September 1851 an das Publikum verkauft und der Rest von 36922 Stück am 10. Dezember 1851 in dem Gebäude der Oberpostdirektion zu Leipzig verbrannt worden.

In Anbetracht dieser hohen Auflage wäre der sehr hohe Preis, welcher heute für gute Stücke dieser Marke gezahlt wird, nicht gerechtfertigt. Er erklärt sich jedoch wohl so, $\mathrm{da} B$ viele mittlere Sammler, die es von vornherein aufgeben, ihre Bremen- oder Oldenburgblätter zu komplettieren, doch stets versuchen werden, die schmerzliche, weil meist einzige Lücke auf dem Sachsenblatt auszufüllen. Zu berücksichtigen ist ferner, daB der rote Dreier als Zeitungsmarke fast ausschlieBlich auf Streifbändern verwandt und deshalb im Gebrauch meist zerrissen wurde. Der Preis für wieder zusammengesetzte oder sonst ausgebesserte Stücke erreicht 
deshalb auch nur den 5. bis 3. Teil der für gute Stücke gezahlten Summen.

Dic Marke mißt $18^{1 / 3} \mathrm{~mm}$ im Quadrat; die kleinen Größenunterschiede erklären sich durch die Eigenart des Stereotypverfahrens, durch welches von dem Originalholzstock die 20 Bleiabgüsse des ganzen Bogens hergestellt wurden, nicht aber durch Dehnung des Papiers, wie manchmal behauptet wird.

Da der Druck in 8 Auflagen erfolgte, ist die rote Farbe der Marke nicht einheitlich, sondern variiert von mattziegelrot bis karmin und kirschrot. Die letzteren beiden Farben sind die selteneren. Das Papier ist ein dünnes Schreibpapier und nicht geglättet.

Als Entwertung war in den ersten Tagen Ferlerstrich vorgeschrieben, später wurden fast ausschließlich runde und rechteckige Ortsstempel verwendet, sehr selten vorkommende Stücke mit Vollgitterstempel oder Nummergitterstempel verdienen höhere Bewertung (s. Abschnitt VII).

Während ihrer eigentlichen Kurszeit war die Marke nur als Streifbàndfrankatur im Gebrauch, während für Briefe auch weiterhin noch Barfrankatur vorgeschrieben war. Nachzügler fanden auch nach dem 1. August 1851 besonders auf Stadtbriefen Verwendung, für die das Porto damals $6 \mathrm{Pf}$. betrug. An sich hatte die rote Dreiermarke bis 31. Dezember 1867 Frankaturgültigkeit.

\section{Fälschungen.}

Die Fälschungen der roten Dreiermarke sind ebenso zahlreich, wie teilweise hervorragend gut ausgeführt. Die ersten Fälschungen stammen schon aus den 50 er Jahren und wurden in Buchdruckausführung von Nürnberg (Zechmeyer), später in Steindruck von Hamburg (Goldner und andere) aus vertrieben. Sehr gute Steindruckfälschungen wurden Ende der 80 er Jahre, von Dresden aus (Elb und Fohl) 
angeboten, sind aber leicht an dem auf echten Marken fast nie vorkommenden Vollgitterstempel zu erkennen. Auch das von Gebrüder Senf seinerzeit herausgegebene Faksimile auf geglättetem Schreibpapier wurde durch Entfernung des Aufdrucks „Falsch" und Ueberstempelung der Rasur „echt" gemacht. Die Stahlstichfälschung (1890) von Thiele, Hannover, ist daran zu erkennen, daß die Schraffur der großen Wertziffer aus Punkten und nicht aus kleinen „3“ besteht. Die beste Fälschung ist der berüchtigte Lichtdruck des Photographen Schröder, der Ende der 70 er Jahre in der Leipziger Lichtdruckanstalt von Naumann \& Schröder ausgeführt und in Zeichnung, Papier und Farbe vom Original kaum zu unterscheiden ist. Man erkennt ihn eigentlich nur daran, daß der Druck, wenn man ihn schräg gegen das Licht hält, vollkommen flach und giatt erscheint, während die im Buchdruçkverfahren hergestellten Originale rauh, an den Druckstellen etwas vertieft und rückseitig durchscheinend sind. Die Farbe des Lichtdrucks ist mehr kirsch- als ziegelrot, und das sehr glatte Papier hat einen leicht bläulichen Schein, während das der Originale mehr gelblich ist.

Daß sich anscheinend auch das Ausland an den Fälschungen beteiligt, schließe ich daraus, daß ich kürzlich aus Paris eine Fälschung mit dem Ortsstempel „Risa“ statt Riesa erhielt.

In allerletzter Zeit werden nach Thier vorzügliche Fälschungen, anscheinend von Sachsen aus, mit Gitterstempel oder mit dem Doppelkreisstempel Leipzig 21. Oct. 50, in der Mitte 5-51/2, vertrleben.

Die nachstehende kurze Beschreibung der

\section{Echtheitsmerkmale}

soll an Hand der vergrößerten Zeichnung dem Sammler die Möglichkeit geben, wenigstens gröbere Fälschungen sofort zurückweisen zu können. Ich rate aber auf jeden Fall, 
Marken, bei denen alle Echtheitsmerkmale stimmen, doch noch entweder meinem Mitarbeiter und Spezialprüfer von Sachsenmarken und Sachsenstempelı

Walter A. Opitz, Netzschkau i. Vogtl.,

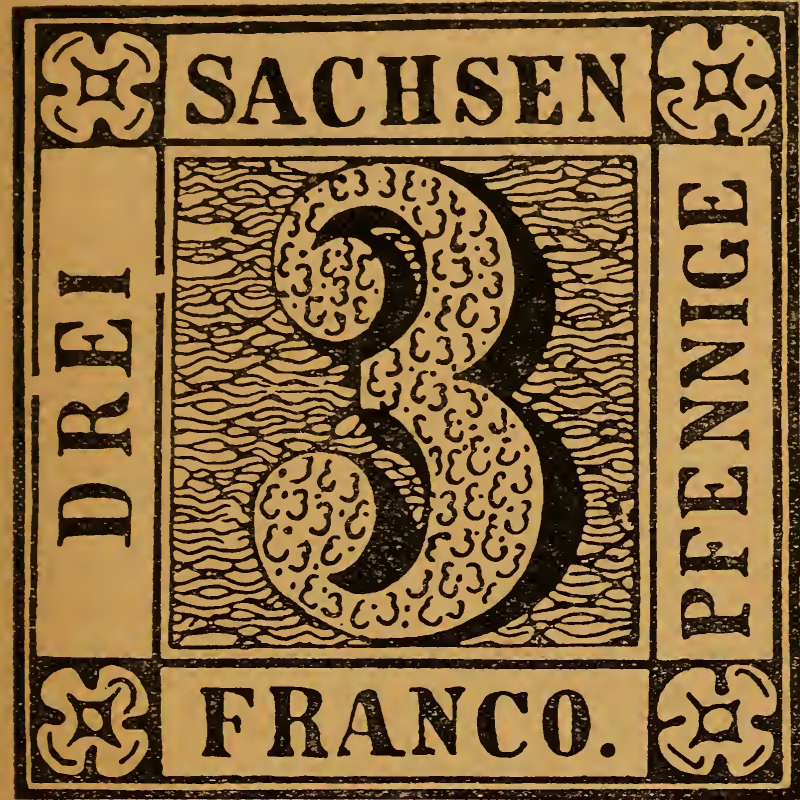

oder einer der amtlichen Prüfungsstellen:

Max Thier, Charlottenburg I, Kaiser-FriedrichStraße 19 oder

A. E. Glasewald sen., Gößnitz (Sachsen-Altenburg) einzusenden und mit deren Prüfungszeichen auf der Rückseite versehen zu lassen.

Die erste Gruppe der Echtheitszeichen ist mit bloßem Auge zu sehen und läßt sich leicht im Gedächtnis behalten; 
stimmen diese Zeichen, so muß man mit Hilfe der Lupe die zweite Gruppe der Kennzeichen prüfen.

I. Gruppe. Die inneren Einfassungslinien sind fast immer in der Mitte zwischen $\mathrm{R}$ und $\mathrm{E}$ oben und fast genau unter dem I unten in dem Worte DREI auf etwa $1 / 4 \mathrm{~mm}$ unterbrochen; die Schraffur des inneren Quadrates zeigt fast genau unter der letzteren Bruchstelle, sowie rechts und links vom oberen Rande der großen Wertziffer zwei, liegenden Ausrufungszeichen ähnliche, und über dem ersten $E$ in PFENNIGE eine hakenförmige kleine weiße Stelle.

Die inneren Verzierungen der Eckrosetten sind ungleichmäBig. Die Rosette links oben hat gleichmäßig auf jedem Blatt innen einen kleinen Halbmond, der in den beiden oberen Blättern etwas zu weit nach rechts steht. In der rechten oberen Rosette fehlt der Halbmond oben links und ist oben rechts in Verlängerung des Sternes in der Mitte nur punktartig angedeutet. Der Rahmen dieser Rosette ist rechts unten auf etwa $1 / 4 \mathrm{~mm}$ unterbrochen. In der Rosette rechts unten fehlt ebenfalls oben links der Halbmond, sowie die beiden rechten Spitzen des Sternes in der Mitte, der Haibmond rechts unten erscheint nur als kürzerer Strich, während die anderen beiden auf einer Seite hakenförmig gebogen sind.. In der Rosette unten links erscheint der linke obere Halbmond nur als schwacher Punkt oder fehlt ganz, während der rechte obere Halbmond stark nach unten verlängert, fast den Rand des Blattes erreicht.

Hinter FRANCO, und zwar nur hinter diesem Wort, steht ein Punkt. Bei dem A in FRANCO fehlt der Fußstrich des linken Balkens fast gänzlich, während der rechte Balkerł einen nach rechts verlängerten Fußstrich hat. Das ganze Wort DREI steht etwa $1 / 5 \mathrm{~mm}$ zu weit nach links.

II. Gruppe. Wenn die in Gruppe I angeführten Merkmale sämtlich vorhanden sind, nimmt man mit Hilfe der Lupe die weitere Prüfung vor. Das Innere der großen 
Wertziffer ist mit 71 kleinen, oft verkrüppelten oder umgekehrten kleinen Drcien und 41 Punkten ausgefüllt. In der Ausfüllung des unteren Punktes der großen Wertziffer stehen eine etwas verkrüppelte und zwei deutliche kleine Dreien fast genau senkrecht übereinander. Das innere Quadrat ist mit dünnen, unregelmäßigen Wellenlinien und etwas dickeren, sich oft kreuzenden Schraffierungslinien bedeckt, die rechts und links über, rechts unter der großen Wertziffer, sowie in der linken unteren Ecke kleine, liegenden Ausrufungszeichen ähnliche weiße Stellen zeigen. Eine etwas kleinere tropfenähnliche weiße Stelle ist ziemlich genau in der Mitte der Marke.

In dem Worte SACHSEN ist das erste $S$ unten, das zweite $\mathrm{S}$ oben offener gehalten; das $\mathrm{N}$ ist rechts unten etwas zu kurz. Das $G$ in PFENNIGE steht etwa $1 / 10 \mathrm{~mm}$ zu niedrig, ebenso das $\mathrm{C}$ in dem Worte FRANCO. Links über dem $O$ dieses Wortes zeigt die Schraffur des inneren Quadrates ein auf der Seite liegendes Kreuz. Der Abstand der äußeren Randlinie ist oben links und unten rechts größer als an den anderen Stellen.

Alle diese Merkmale hat aber die schon erwähnte Schrödersche Lichtdruckfälschung auch; den letzten endgültigen Anhalt gibt deshalb die Druckart, das Papier und die Farbe, die für Nichtkenner nur an der Hand von einwandfreiem Vergleichsmaterial festgestellt werden können. Iclı will auch mit dieser Beschreibung nur dem Durchschnittssammler die Möglichkeit geben, sich vor groben Fälschungen von vornherein $\mathrm{zu}$ schützen. Das endgültige Urteil mag er getrost den schon erwähnten Hernen Opitz, Thier oder Glasewald überlassen, deren Echtheitszeichen auch den etwaigen Verkaufswert der Marke schließlich um mindestens den Betrag der Prüfungskosten steigert. 
III.

\section{Buch- und Kupferdruckausgabe vom 29. Jüli 1851 , im Gebrauch ab 1. August 1851.}

Da die rote Zeitungsmarke von vornherein nur als Notbehelf gedacht war, bekam ihr Drucker Hirschfeld bereits im Juni 1850 den Auftrag, Entwürfe für die endgültigen Briefmarken vorzulegen. Nach langen Verhandlungen gelangte denn auch der Hirschfeldsche Entwurf, nach Verwerfung der sechs Entwürfe des Finanz-Vermessungsdirektors Preßler, für die neue

\section{Kreuzbandmarke 3 Pfennige grün}

mit geringfügigen Ändcrungen zur Annahme, während die weiter geplanten Kreuzbandmarken zu 6 und 18 Pfennig nicht zur Ausführung kamen. Die Marke wurde am 29. Juli 1851 ausgegeben und war zur Freimachung der Kreuzbandsendungen und Stadtbriefe bestimmt. Sie ist vom Juli 1851 bis Januar 1863 von Hirschfeld in 24 Auflagen in einer Gesamtzahl von

gedruckt worden.

$$
\text { 121/4 Millionen Stück }
$$

Durch die große Zahl der Auflagen erklärt sich auch, da B eine ganze Anzahl Abweichungen in der grünen Farbe und in der Färbung des Papiers vorkommen. Die seltenste Farbe ist ein

saftiges Grün bis Dunkelgrün auf weißem Papier, diese Färbung wurde in Zehnerbiocks gedruckt. 


\section{Die Farben}

grün bis blaugrün auf gelblichem Papier, gedruckt in vollen Bogen, kommen ziemlich oft, die hellgelbgrüne Färbung etwas weniger, und die ausgesprochen maigrün bis tiefgelbgrüne Farbe ziemlich selten vor.

Der bei der hohen Auflage der Marke verhältnismäßig sehr hohe Katalogpreis erklärt sich, wie bei der roten Kreuzbandmarke dadurch, daß im Gebrauch die meisten Stücke zerrissen wurden. Die Marken sind sehr eng zusammen gedruckt. Vierseitig vollrandige Stücke sind deshalb höher zu bewerten.

Die wenig vorkommenden Fälschungen der Marke sind grob ausgeführt und deshalb leicht zu erkennen.

Für die höheren Werte dieser Ausgabe erfolgte im September 1850 die Verordnung, daß sie neben der Angabe des Wertes ausschließlich mit dem Bilde des Königs auszustatten seien. Die von Hirschfeld vorgelegten Proben gelangten jedoch nicht zur Annahme, sondern die Entwürfe der Hofbuchdruckerei C. C. Meinhold \& Söhne in Dresden, obgleich Meinhold an den ihm zur Verfügung gestellten Hirschfeldschen Zeichnungen nur geringe Änderungen vorgenommen hatte. Die von Meinhold Ende Mai 1851 vorgelegten Probedrucke in Kupferdruck auf rosaem, gelbem und weißem Papier wurden jedoch nicht genehmigt, sondern am 12. Juni 1851 bestimmt, daB der Druck der Marken zu

$$
\begin{aligned}
& 1 / 2 \text { Neugroschen auf silbergrauem, }
\end{aligned}
$$

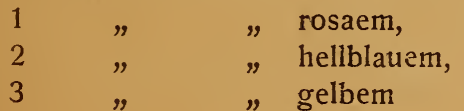

Papier zu erfolgen habe.

Der nach rechts blickende Kopf des Königs Friedrich August erscheint auf glattem schwarzen Gründe, der den 
Marken nach dem tragischen Tode des Königs den Namen „Trauersachsen" eintrug. Tatsächlich zeigt jedoch der Grund eine ganz feine wagerechte Schraffur, die freilich nur bei den ersten Auflagen deutlich zu sehen ist.

Im ganzen wurden von Meinhold gedruckt:

$1 / 2$ Neugroschen schwarz auf silbergrau

vom Juni 1851 bis Oktober 1854 in 17 Auflagen

5 Millionen 100000 Stück.

Im August und September 1851 wurde in Leipzig festgestellt, daB ein Blatt von 120 Stück (12 Zehnerblocks) der $1 / 2$-Neugroschen-Marke versehentlich auf dem hellblauen $\mathrm{Pa}$ pier der 2-Neugroschen-Marke gedruckt und abgeliefert worden war.

63 Stück davon sind an das Publikum verkauft worden. Ein Zehnerstreifen fand sich seinerzeit bei meinem Bankhaus Frege \& Co. vor und wurde von diesem dem verstorbenen Dresdner Sachsen-Spezialisten Dr. Kloss überlassen, wodurch die Marke eigentlich erst als Fehldruck der Sammlerwelt bekannt wurde. Dieser Zehnerblock ist später zerschnitten worden und ein Viererstreifen ging vor kurzen auf einer Auktion für M. 84000 in den Besitz eines Kopenhagener Herrn über.

57 Stück verblieben in den Händen der Post und wurden an das Finanzministerium zurückgeliefert. Dieses hat dann die Marken in den 90 er Jahren das Stück für M. '3.- an Sammler verkauft.

Gebraucht sollen von dem Fehldruck 2 Stücke existieren; ob die Stempel aber echt sind, ist zum mindesten zweifelhaft.

Ich warne ausdrücklich vor gefärbten $1 / 2$-NeugroschenMarken, die fast immer gebraucht angeboten werden, da den Fälschern die ungebrauchten Marken für ihre Kunststücke anscheinend zu teuer sind.

\section{Neugroschen schwarz auf rosa}

vom Juni 1851 bis Oktober 1854 in 17 Auflagen 5 Millionen 700000 Stück. 
2 Neugroschen scliwarz auf hellblau (himmelblau) vom Juni 1851 bis Februar 1852 in 7 Auflagen 700000 Stiick.

Im März 1852 beschwerte sich das Postamt Frciberg, daß das hellblaue Papier der 2-Neugroschen-Marke in grau ausbleiche. Der Drucker crhielt daraufhin die Anweisung, für wcitere Auflagen ein weniger ausbleichendes, dunkelblaues Papier zu verwenden und druckte dann von der

2 Neugroschen schwarz auf dunkelblau vom Mai 1852 bis Oktober 1854 in 9 Auflagen 1 Million 500000 Stück.

Ungebraucht ist diese Marke außerordentlich selten, da die Bestände fast restlos aufgebraucht wurden.

Von der

3 Neugroschen schwarz auf gelb sind vom Juni 1851 bis Oktober 1854 in 16 Auflagen 2 Millionen 350000 Stück

gedruckt worden. Das Gelb kommt in satterer und etwas hellerer Farbe vor.

Der ganze Druckausschuß und die abgenutzten Platten sind s. Zt. von der Behörde vernichtet worden.

Diese Ausgabe wurde in Bogen von 120 Stück, später von 100 Stück gedruckt, zu Blocks von 10 Stück zerschnitten und in Kästchen zu je 200 Stïck verpackt an die Postanstalten geliefert. 
IV.

\section{Kupferdruckausgabe vom Juni 1855 mit Kopf des Königs Johann nach links.}

Nach dem Tode des Königs Friedrich August am 9. August 1854 wurde angeordnet, da $B$ die Freimarken in Zukunft das Bild des neuen Königs Johann tragen sollten. Mit der Gravur des Brustbildes wurde der Münzgraveur Ulbricht beauftragt, während der Druck wieder durch Meinhold, Dresden, im Kupferdruckverfahren erfolgte.

Um die Abstempelung deutlicher in Erscheinung treten zu lassen, wurde der Kopf statt auf glatt erscheinendem, jedoch in Wirklichkeit fein wagerecht schraffiertem schwarzen Grunde, in grob senkrecht schraffiertem Oval angebracht. Gleichzeitig erhielt Meinhold den Auftrag, für die Freimachung der Sendungen nach Ländern, die nicht zum Deutsch-Österreichischen Postverein gehörten, Proben von Freimarken zu 5 und 10 Neugroschen anzufertigen. Durch Verordnung vom 24. April 1856 wurde dann bestimmt, daß die grüne Dreipfennigmarke beizubehaiten sei, die Marken zu 1/2, 1, 2 und 3 Neugroschen in den bisherigen Farben, die neuen Werte $\mathrm{zu}$

5 Neugroschen auf weißem Papier in ziegelrotem, die

10 Neugroschen auf weißem Papier in blauem Druck hergestellt werden sollten. Das blaue Papier der 2-Neugroschen-Marke sollte jedoch etwas lichter gehalten werden. $\mathrm{Da}$ der Drucker sich jedoch nicht allzu genau an die Vorschriften hielt, entstanden verschiedene Abarten, auf die ich später zurückkomme. 
Dic Marken der 3. Ausgabe wurden in folgenden Auflagen gedruckt:

$1 / 2$ Neugroschen schwarz auf silbergrau vom März 1855 bis Januar 1863 in 34 Auflagen

17 Millionen 705000 Stück

1 Neugroschen schwarz auf rosarot

vom März 1855 bis Januar 1863 in 24 Auflagen

17 Millionen 345000 Stück.

Von diesen beiden Marken sind die ersten Auflagen in einer im Michel-Katalog von 1921/22 erstmalig als II. Type mit aufgefülırten Abart gedruckt, die die $1 / 2$ in kleinerer und fetterer Schrift zcigt, während alle drei "i" etwa $1 / 3 \mathrm{~mm}$ kleiner sind.

2 Neugroschen schwarz auf blau, stumpfblau und lichtblau

vom März 1855 bis Januar'1863 in 34 Auflagen

5 Millionen 280000 Stück.

Obgleich dem Drucker die Verwendung eines lichtblauen Papier vorgeschrieben war, ist doch ein großer Teil der Auflagen auf dem ausgesprochen dunkelblauen Papier der dunkelblauen Zweigroschenmarke der 2. Ausgabe gedruckt. Die lichtblaue und die stumpfblaue Farbe wird etwa $30 \%$ höher bewertet. Vorkommende grünlichblaue Stücke sind durch Zersetzung des Gummis entstanden.

3 Neugroschen schwarz auf gelb vom März 1855 bis Januar 1863 in 24 Auflagen 7 Millionen 560000 Stück.

Das Papier ist bei den verschiedenen Auflagen nicht gleichmäßig ausgefallen; man unterscheidet hellgelbe, zitronengelbe, gelbe und dunkelgelbe Stücke.

\section{Neugroschen auf weißem Papier}

ziegelrot, rostbraun, bräunlichrot, karminrot, rotbraun, rostrot vom Februar 1856 bis Januar 1863 in 17 Auflagen 1 Million 90000 Stück, 
und zwar

200000 Stück ziegelrot (feuerrot)

$66200, \quad$ rostbraun (Fehldruck)

825800 " bräunlichrot, karmin, rotbraun, rostrot.

Während die erste Auflage von 200000 Stück eine ausgeprägt feurig-ziegelrote Farbe zeigte, war später bei der Farbenfabrik die Farbe ausgegangen, weshalb Meinhold in Februar 1857 die nächsten 100000 Stück in rostbrauner (dunkelbrauner) Farbe druckte. Die Oberpostdirektion verordnete nun auf eingegangene Beschwerden hin, daB die Bestände dieses

\section{"Fehldruckes zu 5 Neugroschen in dunkelbrauner Farbe"}

an Meinhold zum Umtausch zurückzusenden seien. Von den gedruckten 100000 Stück waren jedoch schon 66200 an das Publikum verkauft, während für den Rest von 33800 Stück von der Druckerei im März 1856 Ersatz geliefert wurde. Diese zurückgelieferten Fehldrucke wurden dann im März 1855 im Gebäude der O.P.D. in Leipzig verbrannt, weshalb ungebrauchte Stücke des Fehldrucks ziemlich selten vorkommen.

Die bei den nächsten 15 Auflagen zu beobachtenden Abweichungen in der Farbe beruhen teilweise auf Schwierigkeiten in der Farbenmischung, teils auf Einwirkungen des Lichtes, des Gummis und der Luft. Eine der letzten Auflagen - wenn nicht die letzte - ist in roströtlicher Farbe auf glasigem Papier gedruckt worden. Diese Abart kommt ungebraucht ziemlich häufig vor, ist dagegen gebraucht nur in wenigen Stücken bekannt.

10 Neugroschen blau auf weißen und glasigem Papier

vom Februar 1856 bis Mai 1861 in 3 Auflagen 250000 Stück.

Wenn auch die Marke nur in 3 Auflagen gedruckt worden ist, kommt doch die blaue Farbe, teilweise auch 
durch Einwirkung des Lichts, in den verschiedensten Abstufungen vom stumpfen Milchblau bis zum leuchtenden satten Blau vor. Die Grundfarben der Auflagen dürften dic folgenden gewesen sein:

1. Auflage 1856 milchblau auf gewöhnlichem Papier

2. " 1859 tiefblau auf gewöhnlichem Papier

3. „ 1861 leuchtend blau auf glasigem Papier.

Das in manchen Katalogen irrtümlich angegebene bläuliche Papier ist dadurch entstanden, daß der Drucker die Kupferplatte nicht sauber gewischt hatte, weshalb das Papier auch außerhalb der Druckstellen einen bläulichen Schein bekommen hat.

Die 2. und besonders die 3. Auflage ist gebraucht viel seltener, während die letzte Auflage auf glasigem Papier ungebraucht häufiger vorkommt, weil bei Außerkurssetzung noch Bestände bis etwa 20000 Stück vorhanden gewesen sein sollen.

Obgleich die Marke nur auf Briefen verwendet wurde und deshalb vielfach erhalten blieb, scheint mir auch die neueste stark erhöhte Preisnotierung des Michel-Katalogs noch zu niedrig zu sein, wenn man berücksichtigt, daß die rote Dreiermarke in doppelter Auflage gedruckt worden ist.

Die Marken zu 5 und 10 Groschen gelangten erst am 24. April 1856 zur Ausgabe, seit welchem Datum auch die Briefe nach Ländern außerhalb des Deutsch-Österreichischen Postvereins mit Marken freigemacht werden konnten.

Von der $1 / 2$ Neugroschen gibt es ein durch Schmitzdruck entstandenes Kuriosum 11/22 Neugroschen.

Da die Druckerei Meinhold damals wenig sorgfältig gearbeitet hat, wurden von vielen Postämtern Ausschußdrucke mit weißen Stellen, Falten usw. zurückgeliefert. Die Bestände solcher Marken wurden erst 1918 verkauft und werden bei der heute grassierenden Spezialisierungswut weiter Kreise - ich erinnere nur an die in sonst guten 
Katalogen aufgenommenen Druckzufälligkeiten der neueren bayrischen Sammler-Ausbeutungsserien - auch sicher ihre Käufer finden. Ich halte eine höhere Bewertung derartigen Druckausschusses für nicht gerechtfertigt, denn der Sammler soll doch schlieBlich Verkehrsdokumente sammeln und nicht Makulatur. - Nicht viel besser sind die bei allen Werten dieser Ausgabe vorkommenden sogenannten

\section{Doppeldrucke,}

die weiter nichts sind, als beim Kupferdruckverfahren manchmal an den Rändern in Erscheinung tretende Schmitzdrucke, die von einer sauber arbeitenden Druckerei auch nicht mit abgeliefert worden wären. - An

\section{Fälschungen}

von Marken dieser Ausgabe kommen fast nur reichlich plumpe Steindruckfälschungen vor, die auch von ungeübteren Sammlern an dem glatten Druck und den abweichenden grellen Farben zu erkennen sind. - Die gute Fälschung der 10-Neugroschen-Marke des bekannten Dreiersachsenfälschers Schröder ist auch in Lichtdruck ausgeführt, jedoch heute fast noch seltener als die echte Marke.

Die geplante Ausführung der Marken dieser Ausgabe in Buchdruck kam nicht mehr zustande, da auf Anregung des preußischen General-Postamts die entsprechenden Werte der Marken der verschiedenen, dem Postverein angehörenden Länder in gleichen Farben erscheinen sollten, was nunmehr Veranlassung zu einer ganz neuen in Buch- und Prägedruck ausgeführten Wappenausgabe gab.

Gegenüber bisher geäußerten Zweifeln, daß die Marken zu 5 und 10 Neugroschen auf kleinen Postämtern nicht erhältlich waren, konnte festgestellt werden, daB die kleine am 31. Dezember 1861 aufgehobene Postexpedition Wildenthal 97 Stück der Zehngroschenmarke an die Oberpostdirektion zurückgab. 
V.

\section{Ausgabe in Buch- und Prägedruck, Wappen- zeichnung, gezähnt 13, vom 1. Juli 1863.}

Die Ausführung dieser Ausgabe, für welche die Adlermarken Preußens als Vorbild dienten, sollte laut Bekanntmachung der Oberpostdirektion in Leipzig auf dem Wege der Submission erfolgen. Von den Entwürfen der zahlreichen, an dem Wettbewerb teilnehmenden Firmen gefielen am besten die Proben der Leipziger Druckerei Giesecke \& Devrient, die dann auch im Februar 1863 endgültig den Auftrag erhielt, sechs verschiedene Werte in buntem

Buch- und Prägedruck auf weißem Papier herzustellen, wobei laut $\S 4$ des Vertrages ,die Marken an den Umfassungsseiten dergestalt fein zu durchlöchern sind, da $\beta$ sie ohne Benutzung eines schneidenden Instrumentes leicht von einander abgetrennt werden können". - Im ganzen wurden von dieser Ausgabe gedruckt:

3 Pfennige blaugrün, gelbgrün, hellgrün, smaragd, moosgrün vom Mai 1863 bis August 1867 in 17 Auflagen 10 Millionen 350000 Stück.

Die auch gebraucht recht oft vorkommende blaugrüne Farbe war in den Katalogen überteuert und ist im Michel 1921/22 sehr richtig etwas herabgesetzt worden.

$1 / 2$ Neugroschen rotorange, trüborange, orangegelb vom Mai 1863 bis August 1867 in 17 Auflagen

17 Millionen 100000 Stück. 
Durch Schwierigkeiten bei der Farbmischung sowie teilweise auch durch Einwirkung des Lichtes und des Gummis sind unzählige Farbabarten entstanden, deren genaue Schematisierung unmöglich sein dürfte. Nachstehend gebe iclı in chronologischer Reihenfolge die hauptsächlichsten Farbengruppen:

A. rotorange $(1863-67)$

a) mennigrot (1863-64), b) hellfleischrot

B. trüborange (1866-67)

a) bräunlichorange (terracotta bis lederfarben)

C. orangegelb (1867)

a) gelb, hellgelb, b) kanariengelb, apfelsinengelb,

c) hellzitronengelb.

Die unter $\mathrm{C}$ a-c aufgeführten Farben sind sehr selten. Die kanariengelbe Farbe kommt gestempelt sehr selten vor, wälrend die zitronengelbe gebraucht bisher überhaupt noch nicht bekannt ist.

1 Neugroschen rosa, hellrosa, karminrosa bis lilarosa

vom Mai 1866 bis August 1867 in 17 Auflagen 15 Millioneil 175000 Stück.

2 Neugroschen blau, hellblau, tiefblau, preußischblau bis ultramarin

vom Mai 1863 bis August 1867 in 16 Auflagen 4 Millionen 780000 Stück.

3 Neugroschen braun, gelbbraun, hellrehbraun, rötlichbraun, kaffeebraun

vom Mai 1863 bis August 1867 in 17 Auflagen

5 Millionen 870000 Stück.

5 Neugroschen lila

vom Mai 1863 bis Mai 1867 in 15 Auflagen

1 Million 200000 Stück.

Von dieser Marke gibt es nicht weniger als 8 Farbabarten und einen nicht in den Verkehr gekommenen, im Leipziger Postdirektionsgebäude im März 1867 verbrannten röt- 
lichvioletten Fehldruck, ganz abgesehen von deı vielen durch Einwirkung des Lichtes und des Gummis entstandenen Spiclarten dieser Marke.

Es sind zu unterscheiden:

$$
\begin{array}{cll}
\text { Auflagen } & \text { is63 } & \text { blaugrau } \\
\text { " } & 1864-65 & \text { grünlichblau } \\
\text { " } & 1866 & \text { lila, rotlila } \\
, \quad & 1866 & \text { steingrau } \\
\text { " } & 1867 & \text { brämilichlila, graulila, blaugrau. }
\end{array}
$$

Während die letzten 3 Farben gebraucht die teuersten sind, kann man die graulilae Farbe aus verkauften Restbeständen auch heute noch verhältnismäßig billig und die grünlichblate Abart nicht viel teurer erwerben.

Sämtliche Werte der Wappenausgabe kommen auch auf sehr dünnem, durchsichtigem oder auch sehr dickem Papier vor.

Mit dem Erscheinen neuer Ausgaben wurden die Marken früherer Ausgaben nicht außer Kurs gesetzt, so daß alle Sachsenmarken bis 31. Dezember 1867 verwendet werden konnten.

Mit dem Übergang der sächsischen Postverwaltung an den Norddeutschen Postbezirk und Einführung der Marken dieser Post kamen die sächsischen Marken mit dem

\section{Januar 1868}

außer Kurs. Die Restbestände der ungebrauchten Marken sind dann in späteren Jahren vom Finanzministerium veräußert worden.

Wann und zu welchen Preisen die Restbestände verkauft wurden, läßt sich nicht mehr genau feststellen. Die noch in großen Mengen vorhandenen Werte der Freimariken und Ganzsachen wurden Ende der 60 er Jahre für ganze 3 Taler für den Zentner verkauft. Etwas teurer waren die vorhandenen 20 Stück rote Dreiermarken und 57 Stück hellblaue 
Fehldrucke, die in den $90 \mathrm{er}$ Jahren für 3 Mark für jedes Stück an Sammler abgegeben wurden.

Interessant ist die "Preisliste", welche das Finanzministerium im Jahre 1891 auf Verlangen versandte. Nach dieser kostete die grüne Dreiermarke von 185130 Pfennige, von der Ausgabe König Johann die Werte von $1 / 2-5$ Neugroschen den dreifachen Nennwert und die 10-Neugroschen 15 Mark.

Am billigsten war die Wappenausgabe, die man damals zu

10 Pfennig für die 3 Pfennigmarke

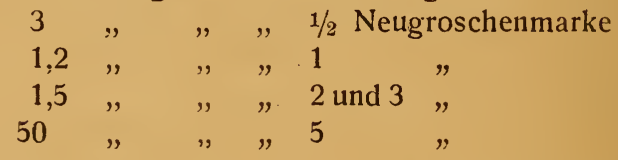

beziehen konnte. Später sind dann die Preise etwas erhöht worden. Die letzten Bestände, darunter auch viel Makulaturdrucke, sind erst 1918 verkauft worden.

VI.

Laut der bei Einführung der Frankokuverts getroffenen Bestimmung (Bekanntmachung vom 23. Juni 1859) durften „aus verdorbenen Franco-Couverts ausgeschnittene, noch nicht entwertete Stempel als Franco-Marken verwendet werden". Sie haben für Briefmarkensammler jedoch nur Sammelwert, wenn der Stempel auf den Brief oder das Briefstück übergeht und werden teilweise hoch bewertet. 


\section{VII.}

\section{Die sächsischen Francostempel, Entwertungsstempel und ihre Bewertung für den Sammler.}

1.

Bereits lange Zeit vor Einführung der sächsischen Briefmarken waren sächsische Poststempel im Gebrauch, mit denen die Briefe nach Erlegung der Barfrankatur abgestempelt wurden. Es lassen sich solche sächsische Stempel bis zur napoleonischen Zeit zurück feststellen.

Einige dieser sogenannten Vorläufer sind verhältnismäßig selten. Die vorphilatelistischen Briefe werden jedoch meist nur von großen Spezialsammlern systematisch gesammelt; ich bewerte sie je nach Alter, Erhaltung und Ort nur auf 1-10 Mark. Man muB bei solchen Briefen nicht nur die Seltenheit in Betracht ziehen, sondern auch die schon erwähnte Tatsache, daß nur wenige Spezialsammler als Käufer in Frage kommen.

Alte sächsische Postquittungen aus dem 18. Jahrhundert dürften etwa gleich hoch zu bewerten sein.

Bei Einführung der

$$
\text { roten Dreipfennigmarke }
$$

wurde in der Verordnung vom 22. Juni 1850 bestimmt, daß auf Kreuzbandsendungen innerhalb Sachsens die Marken mit starken

$$
\text { schwarzen Federstrichen }
$$

kreuzweise so durchstrichen werden sollten, daB die Striche auf beiden Seiten der Marke das Papier des Kreuzbandes 
berührten. Daneben mußte noch der Ortsstempel angebracht werden.

Da diese an und für sich sehr seltene Entwertung nur auf voll erhaltenen Kreuzbändern mit nebenstehendem Ortsstempel als echt nachgewiesen werden kann, werden lose Stücke mit Tintenstrichentwertung wenig geschätzt.

Die Federstrichentwertung wurde jedoch schon am 5. Juli 1850 durch Verordnung wieder aufgehoben und bestimmt, daß Kreuzbandmarken

„vorläufig mit dem Ortsstempel in schwarzer Farbe“ zu entwerten seien.

Da die Einführung der geplanten besonderen Entwertungsstempel sich sehr verzögerte, kommt die rote Dreiermarke fast ausschließlich mit den zumeist schon in der vorphilatelistischen Zeit im Gebrauch gewesenen Ortsstempeln in runder, eckiger und gezackter Form vor.

Die durch Verordnung vom 11. März 1852 eingeführten Vollgitterstempel aus Messing, sowie die erstmalig im Dezember 1852 erscheinenden stählernen Nummergitterstempel können, da die rote Dreiermarke zur Zeit der Einführung dieser Stempel bereits durch die Ausgabe in grüner Farbe ersetzt war, naturgemäß nur sehr selten auf Nachzüglern vorkommen.

Die verschiedenen Stempel auf der Dreiermarke dürften wie folgt zu bewerten sein:

Zuschlag zu dem jeweiligen Katalogpreis: Federstrichentwertung auf ganzem Kreuzband. $150 \%$ runder Ortsstempel . . . . . . . . . . . . $-0 \%$ rechteckiger Ortsstempel . . . . . . . . . . $10 \%$ Altenburger Ortsstempel ohne Einfassung (s. Abb.) $10 \%$ Altenburger Posthornstempel . . . . . . . $1000 \%$ Vollgitterstempel . . . . . . . . . . . $50 \%$ Nummerngitterstempel . . . . . . . . . . . $100 \%$ Dresdner Stadtpoststempel . . . . . . . . . $10 \%$ Stempel in blauer Farbe. . . . . . . . $10-25 \%$ 
$\begin{array}{r}\text { KLINGEN } \\ \text { 20FEB. }\end{array}$
$\mathcal{L}_{\text {eipzig }}$

3

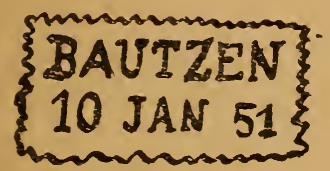

5
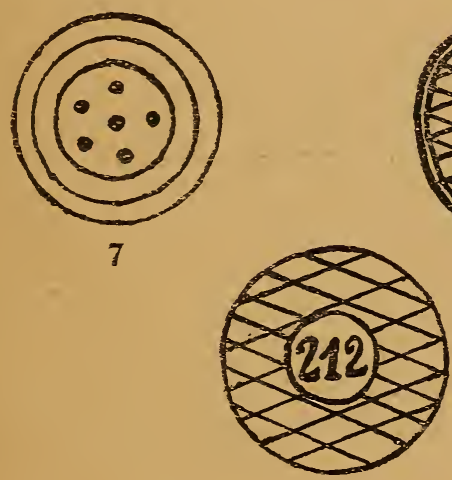

9

4

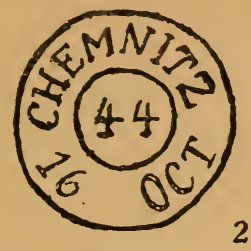

ALTENBURG $12 \mathrm{MAI}$.
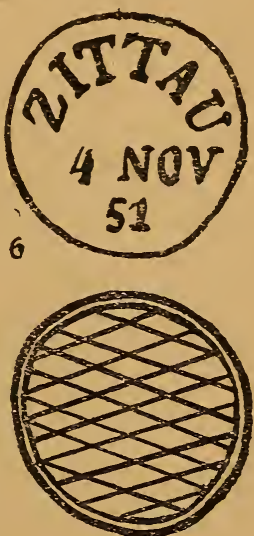

8

$1,2,4-6$ vorphilatelistische Stempel, teilweise bis etwa 1860 in Gebrauch. 3 Zierschriftstempel Leipzig. 7 und 8 Chemnitzer Ver. suchsstempel. 9 der seltenste Nummergitterstempel. 


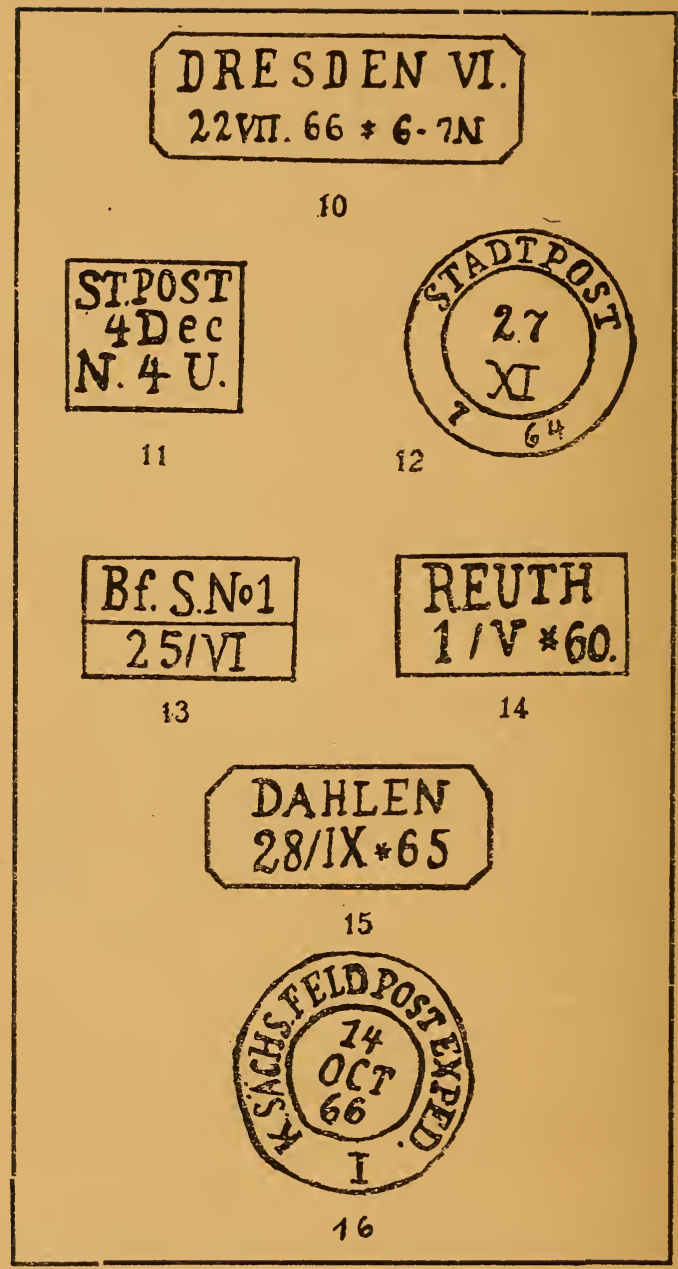

10 Dresdner Langstempel. 11-12 Stadtpoststempel. 13-15 Stempel der Briefsammlungen. 16 Feldpoststempel. 
3.

Dreipfennigmarke grün von 1851.

Bei der langen Kursdauer dieser Marke kommen alle möglichen Entwertungsarten vor. Besondere Beachtung verdienen: Federstrich-, rote und blaue Entwertungen, sowie der alte, fast quadratische Dresdner Stadtpoststempel (s. Abb.) und der Einzeiler „Leipzig“ (s. Abb.) in Zierschrift.

4.

\section{Kopfausgabe König Friedrich August von 1851.}

Die Marken dieser Ausgabe sind meist durch Vollgitterstempel entwertet worden. Weniger kommen die Ortsstempel vor, welche auf der dunkelblauen Zweigroschenmarke sogar sehr selten sind, während dagegen Nummerngitterstempel auf der hellblauen Zweigroschenmarke ebenso seiten vorkommen. Sehr gesucht sind auch farbige Entwertungen.

Nummerngitterstempel kommen bis Nr. 59 öfter, bis Nr. 133 seltener und höhere Nummern nur auf Nachzüglern sehr selten vor. Block- und Zierschriftstempel sowie der Chemnitzer Sechspunkt-Versuchsringstempel (s. Abb.) bilden auch auf diesen Marken gesuchte Seltenheiten. Auch der ebenfalls in Chemnitz vom Dezember $1851 \mathrm{ab}$ einige Zeit in Gebrauch gewesene Vollgitterstempel mit doppelter Außenlinie (s. Abb.) ist noch beachtenswert als einziger Stempel dieser Art.

5.

Kopfausgabe König Johann von 1855.

Die häufigste Entwertungsart ist bei den Marken dieser Ausgabe der schwarze, seltener farbige Nummerngitterstempel. Da die Ausgabe dieser Stempel erst nach und nach erfolgte - die Ausgabedaten sind in den später folgenden Preistafeln bei den einzelnen Postämtern ange- 
geben -, findet man in den Jahren 1855/56 außer der Ortsstempeln auch noch bei mehr als 100 Poststellen auch den schon auf der vorhergehenden Ausgabe im Gebrauch gewesenen Vollgitterstempel. Beachtung verdienen aber nur Stücke auf ganzen Briefen mit danebenstehendem deutlichen Ortsstempel. Ziemlich selten auch auf losen Marken sind die Vollgitterstempel nur auf den erst 1856 ausgegebenen Werten zu 5 und 10 Neugroschen. Vorkommende Stücke dürften oft nachträglich angebrachte falsche Stempel haben.

Die Zuteilung der Nummerngitterstempel erfolgte:

Nr. 1 und 2 im Dezember 1852

3 bis 61 bis Mitte 1855

" 62 bis 85 am 13. August 1855

„ 86 bis 108 am 3. November 1855,

die folgenden Nummern bis 177 in der Zeit vom 1. Januar bis 12. Juli 1856. Bei den restlichen Nummern von 178 bis 220 ist das Ausgabedatum in den nachstehenden Preistafelñ angegeben.

Häufig findet man auf dieser Ausgabe auch schwarze, seitener farbige Ortsstempel, da im Februar 1859 die frühere Verordnung dahin geändert wurde, daß Kreuzbandsendungen

\section{nur mit Ortsstempel}

und dann laut Verordnung vom 22. Dezember 1859 auci $\mathrm{s}$ äm tliche Sendungen so entwertet werden durften. Neue Nummernstempel wurden ab 1862 nicht mehr angefertigt; die vorhandenen Stempel sollten aber laut nachstehender Verordnung bis zur Abnutzung in Gebrauch bleiben.

Verordnung vom 22. 12. 1859:

Die K.O.P.D. hat beschlossen, die dermalen im Gebrauch befindlichen Entwertungsstempel nach erfolgter Abnutzung nicht wieder zu erneuern, sondern die Entwertung 
mittels Aufdrückung des Aufgabestempels auf die Frankomarken resp. auf die Couvertstempel bewirken zu lassen. Die regelmäßige Entwertung der Marken mittels des Aufgabestempels darf von keiner Postanstalt früher bewirkt werden, als bis die dermaligen Entwertungsstempel völlig abgenutzt sind, einer der Inspektionsbeamten sich hiervon persönlich überzeugt und die fernere Verwendung des Entwertungsstempels als ungeeignet bezeichnet hat.

Selten kommen auf Marken dieser Ausgabe auch Stempel fremder Posten (Thurn und Taxis, Preußen) usw. vor.

6.

\section{Wappenausgabe von 1863 .}

Da wie oben erwähnt seit 1862 keine neuen Nummernstempel mehr ausgegeben wurden, kommen auf dieser Ausgabe Ortsstempel etwas häufiger vor, wie Nummerngitterstempel. Geschätzt sind auch auf dieser Ausgabe farbige Entwertungen, ferner Zufallsstempel, der blaue Eibenstocker Gerichtsstempel mit dem sächsischen Wappen, der Leipziger Hufeisenstempel und die Stempel fremder Posten.

Aucl nach dem 1. Januar 1868 blieben viele Nummernstempei neben den bisherigen Ortsstempeln im Gebrauch. Von ersteren sind auf Marken des Norddeutschen Postbezirks etwa 100 Nummern bekannt und teilweise sehr gesucht, während Ortsstempel auf diesen Marken und auf Marken des Deutschen Reiches sehr oft vorkommen, denn der letzte dieser Stempel (Mohorn) ist erst während des Weltkrieges außer Gebrauch gekommen.

Von Nummernstempeln auf Marken des Deutschen Reiches ist bisher nur die $\mathrm{Nr} .7$ bekannt.

\section{7.}

Die Ortsstempel hatten meist runde Formen, es kommen jedoch auch viele Langstempel mit und ohne Einfassung vor. 
In

Dresden und Leipzig

waren außerdem noch

Stadtpoststempel (s. Abb.),

die in den nachstehenden Preistafeln genauer angegeben sind, im Gebrauch. Sie kommen jedoch meist nur als Aufgabestempel in roter Farbe auf Briefen neben den mit den Nummernstempel entwerteten alten sächsischen Marken vor.

8.

Einige Posthilfsstellen hatten besondere Langstempel ohne Stundenangabe (s. Abb.) für die bei ihnen eingerichteten

\section{Briefsammlungen.}

Diese Stempel waren aber laut Vorschrift nur auf der Siegelseite des Briefumschlages anzubringen, während die Marken mit dem Stempel der übergeordneten Postanstalt entwertet werden muBten. Die im Bestellbezirk der betreffenden Briefsammlung verbleibenden Briefe konnten auch mit diesem Stempel vorderseitig entwertet werden.

Die nachstehenden Tabellen bringen nicht nur - von Zufallsstempeln abgesehen - eine Aufstellung wohl fast aller der Sachsenforschung bekannten Orts-, Gitter- und Nummernstempel; sondern auch zum ersten Male ungefälıre Preisangaben, die jedoch nicht unbedingt maßgebend sein sollen, sondern nur den Seltenheitsgrad der einzelnen Stempel im Verhältnis zu einander anzeigen sollen, wie er auf Grund langjähriger Beobachtungen von mehreren Seiten festgestellt werden konnte. 
Die Nummernstempel (Halbgitterstempel) in schwarzer Farbe auf den Briefmarken von Sachsen (Sachsen-Altenburg).

\begin{tabular}{|c|c|c|c|}
\hline & Name des Postamtes & \multicolumn{2}{|c|}{$\begin{array}{l}\text { Zuschlag zum Kata } \\
\text { logpreis der Marke! } \\
\text { Ausgabe } \\
\text { Johann | Wappen }\end{array}$} \\
\hline & Dresden (Hofpostamt) & & - \\
\hline & $\begin{array}{l}\text { Leipzig (Obe } \\
\text { do. in rote }\end{array}$ & 10.- & 10.- \\
\hline 3 & $\begin{array}{c}\text { Dresden-Neustadt . } \\
\text { do. Bahnhof . . }\end{array}$ & -.50 & -.50 \\
\hline 4 & Leipzig, Bahnhof . . & -.25 & 25.- \\
\hline & Leipzig--Ho & -50 & 1.50 \\
\hline & den, Zugpost & -.75 & 1.50 \\
\hline 7 & $\begin{array}{l}\text { a, Zugpost, große ,7“" } \\
\text { a-Zwickau, kleine }, 7^{“}\end{array}$ & $\begin{array}{l}-.75 \\
1 .-\end{array}$ & $1 .-$ \\
\hline 8 & 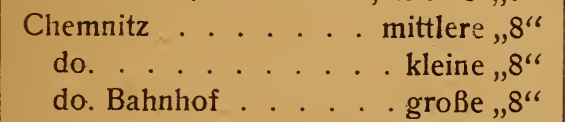 & $\begin{array}{r}-.25 \\
-.50 \\
1.50\end{array}$ & $\frac{-.25}{-}$ \\
\hline 9 & 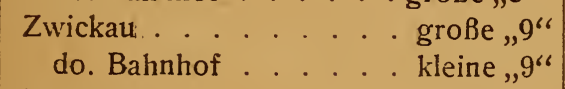 & 1.- & $\frac{-}{1.50}$ \\
\hline 10 & Annaberg . . & - & - \\
\hline 11 & & - & - \\
\hline 12 & Freiberg in Sachsen ....... & - & - \\
\hline 13 & $\begin{array}{c}\text { Schneeberg, ab Januar } 1861 \text { Schnee- } \\
\text { berg-Neustädtel } \ldots \ldots . . . . .\end{array}$ & -.25 & -.25 \\
\hline 14 & 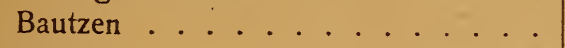 & - & - \\
\hline 15 & $\begin{array}{l}\text { Zittau } \\
\text { do. Bahnhof . }\end{array}$ & $-\overline{-.50}$ & $-\overline{-.75}$ \\
\hline
\end{tabular}

Anmerkung: Von Nr. 1-13, 15, 17-22, 24-25 gibt es verschiedene geringfügig von einander abweichende Typen. 


\begin{tabular}{|c|c|c|c|}
\hline 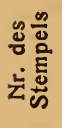 & Name des Postamtes & \multicolumn{2}{|c|}{$\begin{array}{c}\text { Zuschlag zum Kata. } \\
\text { logpreis der Marken } \\
\text { Ausgabe }\end{array}$} \\
\hline 16 & Borna . . & -.25 & -.50 \\
\hline 17 & Glauchau & - & - \\
\hline 18 & Grossenhain . . . . . . . . & - & - \\
\hline 19 & .......... & - & - \\
\hline 20 & Oschatz ........ & -.25 & -.25 \\
\hline 21 & Pirna . . . . . . . . . & - & - \\
\hline 22 & Reichenbach (im Vogtland). . . . & - & - \\
\hline 23 & Rochlitz . . . . . . . & -.25 & -.25 \\
\hline 24 & 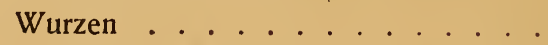 & - & - \\
\hline 25 & Kötschenbroda ab $1.7 .54 \ldots . .$. & -.75 & 3.- \\
\hline 26 & Deutsch-Einsiedel, ab 1.7.54... & 1.50 & 1.50 \\
\hline 27 & Hartenstein, ab 1. 10.54 . . . . & 1.50 & 1.50 \\
\hline 28 & Neukirchen b. Chemnitz, ab 1.10.54. & 2.50 & 2.50 \\
\hline 29 & Wildenfels, ab 1.10.54. . . . . & 1.- & 1.- \\
\hline 30 & Adorf . . . . . . . . & -.75 & -.75 \\
\hline 31 & Auerbach (im Vogtland) . . . . . & -.50 & -.50 \\
\hline 32 & Camenz . . . . . . . . . & -.50 & -.50 \\
\hline 33 & Colditz . . . . . . . . & -.50 & 1.- \\
\hline 34 & Crimmitschau . & -.25 & 3.- \\
\hline 35 & Döbeln ..... . & -.25 & -.25 \\
\hline 36 & $\begin{array}{l}\text { Eibenstock } \\
\text { do.Gerichtsstempel mit sächsischem } \\
\text { Wappen in blauer Farbe.... }\end{array}$ & -.50 & -.75 \\
\hline 37 & Frankenberg in Sachsen . . . . . . & -.50 & -.50 \\
\hline 38 & $\begin{array}{l}\text { Lichtenstein, ab November } 1860 \text { Lich- } \\
\text { tenstein-Callnberg } \ldots . . . . .\end{array}$ & -.50 & -.50 \\
\hline 39 & Dohna, ab $1.3 .55 \ldots . .$. & 2.50 & 2.50 \\
\hline 40 & Glashütte, ab 1.3.55. . . . . & 1.50 & 1.50 \\
\hline 41 & Lauenstein in Sachsen, ab 1.3.55. & 1.- & 1.- \\
\hline 42 & Mülsen St. Jacob, ab 1.4.55 . . . & 1.50 & 1.50 \\
\hline
\end{tabular}




\begin{tabular}{|c|c|c|c|}
\hline 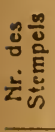 & Name des Postamtes & $\begin{array}{c}\text { Zuschlag } \\
\text { logpreis } \\
\text { Au } \\
\text { Johann }\end{array}$ & $\begin{array}{l}\text { Kum Kata- } \\
\text { er Marken } \\
\text { abe } \\
\text { Wappen }\end{array}$ \\
\hline 43 & Weesenstein, ab 1.4 .55 & 4.- & 5.- \\
\hline 44 & Grimma ........ & -.25 & -.25 \\
\hline 45 & Löbau in Sachsen & -.25 & -.25 \\
\hline 46 & Plauen ..... . & -.25 & -.25 \\
\hline 47 & Waldheim & -.25 & -.75 \\
\hline 48 & Bischofswerda & --.25 & -.25 \\
\hline 49 & Hainichen $\ldots \ldots \ldots$ & -.50 & -.75 \\
\hline 50 & $\begin{array}{l}\text { Hohenstein, ab November } 1858 \text { Hohen- } \\
\text { stein-Ernstthal . . . . . . . }\end{array}$ & -.50 & -.50 \\
\hline 51 & Leisnig . . . . . . . . . . . & -.25 & -.25 \\
\hline 52 & Limbach. & -.25 & -.25 \\
\hline 53 & Meerane. . & -.25 & -.25 \\
\hline 54 & Mittweida & -.25 & -.25 \\
\hline 55 & Penig ... & -.50 & -50 \\
\hline 56 & Ronneburg & -.50 & -.50 \\
\hline 57 & Rosswein. & -.50 & -.50 \\
\hline 58 & Werdau . . & -.25 & -.25 \\
\hline 59 & Zschopau . . . . . & -.25 &.- .25 \\
\hline 60 & Elsterberg, ab $1.7 .55 \ldots \ldots$ & 1.- & 1.- \\
\hline 61 & Harthau bei Chemnitz, ab 1.8.55 & 1.50 & 2.- \\
\hline 62 & Burgstädt $\ldots \ldots \ldots \ldots$ & -.50 & -.50 \\
\hline 63 & Dippoldiswalde & -.50 & -.50 \\
\hline 64 & Eisenberg . . & -.50 & -.50 \\
\hline 65 & Herrnhut . . . & -.75 & -.75 \\
\hline 66 & Johanngeorgenstadt & -.50 & 2.- \\
\hline 67 & Kahla ...... & -.50 & -.50 \\
\hline 68 & Königsbrück & 1.- & 1.- \\
\hline 69 & Königstein . & 1.- & 1.- \\
\hline 70 & Lengenfeld & -.75 & -.75 \\
\hline 71 & | Lössnitz . . . . & -.50 & -.50 \\
\hline
\end{tabular}




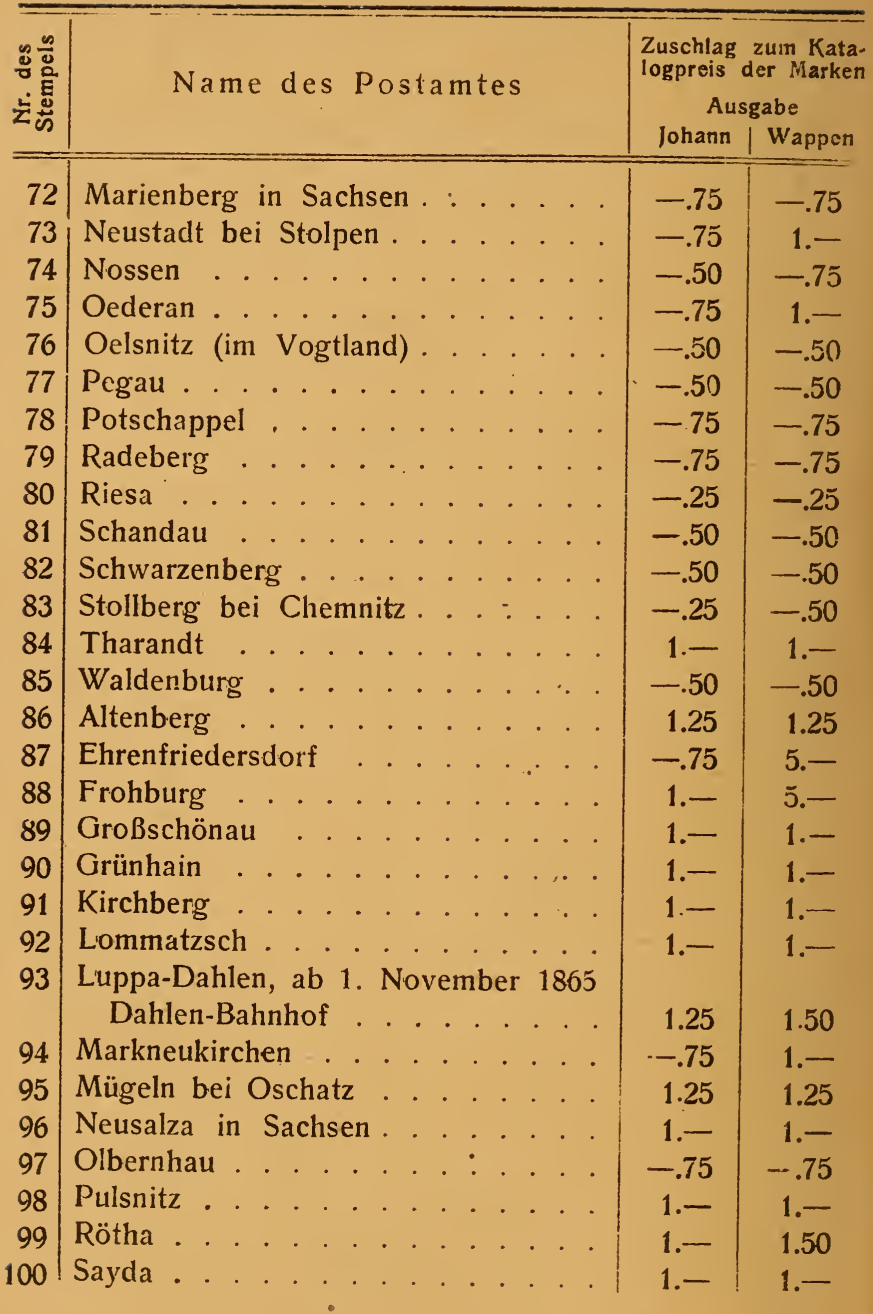




\begin{tabular}{|c|c|c|c|c|}
\hline \multirow{2}{*}{ 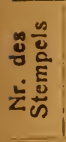 } & \multirow{2}{*}{\multicolumn{2}{|c|}{ Name des Postamtes }} & \multicolumn{2}{|c|}{$\begin{array}{c}\text { Zuschlag zum Kata } \\
\text { logpreis der Marker } \\
\text { Ausgabe }\end{array}$} \\
\hline & & & & Wappen \\
\hline & & . & & .25 \\
\hline & CImom & . & 10 & 1.25 \\
\hline & Scliönhayda (Schönheide) . & $\theta^{\circ}$ & -.75 & -.75 \\
\hline & Sebnitz . . . . . . & . . . & -.75 & -.65 \\
\hline & Strehla & . . & 1.- & 1.- \\
\hline & . . . . . . . . &. & 1.- & 1.- \\
\hline & Vermsdorf & . & 1.- & 1.- \\
\hline & Wilsdruff . . . . . . . & . & 1.- & 1.- \\
\hline & hselburg, ab $1.1 .56 \ldots$ & . & & 1.50 \\
\hline & (2 Typen) & . . & - & -.50 \\
\hline & nstadt (in Sachsen) . . . . & . . & & 1.50 \\
\hline & Bodenbach . . & . . & & 10.- \\
\hline & ch.... & . . & & 1.50 \\
\hline & Eybau . . . . & . . & 1.- & 1.- - \\
\hline & Falkenstein . & . . & 1.- & 1.- \\
\hline & . . . . . & . . & 1.- & 1.- \\
\hline & . . . & . . & 1.- & 1.- \\
\hline & walde . . . . . & . & 1.- & 1.- \\
\hline & Grüna b. Chemnitz, ab 1.1 .54 & . & 1.50 & 1.50 \\
\hline & adt, ab $1.4 .52 \ldots$ & . & 1.- & 1.- \\
\hline & Klingenthal . . . . . . & . . & 1. - & 1.- \\
\hline & gk . . . . . & . . & 1.- & 1.- \\
\hline & Lengefeld im Erzgebirge . . & & 1.- & 1.- \\
\hline & Lucka bei Altenburg. . . . & . $\cdot$ & 1.25 & 1.25 \\
\hline & Neugersdorf in Sachsen . . . & & 1.25 & 1.25 \\
\hline & Radeburg . . . . . & 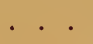 & 1.50 & 1.50 \\
\hline & Roda . . . . . . . & & 1.- & 1.- \\
\hline & $\begin{array}{l}\text { Scheibenberg. . . . . kleine } \\
\text { do. . . . . . . große }\end{array}$ & Ziffer & $\begin{array}{l}3 .- \\
1.25\end{array}$ & 2. \\
\hline & ......... & & 1.50 & 1.50 \\
\hline
\end{tabular}




\begin{tabular}{|c|c|c|c|}
\hline 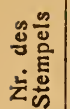 & Name des Postamtes & $\begin{array}{r}\text { Zuschlag } \\
\text { logpreis } \\
\text { Aus }\end{array}$ & $\begin{array}{l}\text { Uum Kata- } \\
\text { er Marken } \\
\text { labe }\end{array}$ \\
\hline & & Johann & \\
\hline 30 & Thum . . . & 1.- & 1.- \\
\hline 131 & Wolkenstein . & 1.25 & 1.25 \\
\hline 132 & 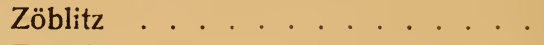 & 1.50 & 1.50 \\
\hline 133 & Zwenkau . . . . . . . & 1.- & 1.- \\
\hline 134 & Wehlen, ab 1.4.56... & 2.50 & 3.- \\
\hline 135 & Ostrau, ab 1.7.56... & 2.- & 2.- \\
\hline 136 & Geyer, ab 15.7.56 . . . . . & 1.25 & 1.25 \\
\hline 137 & Brand bei Freiberg, ab 1.8.56... & 1.50 & 1.50 \\
\hline 138 & Brandis, ab 1.8.56 . . . . . & 1.75 & 1.75 \\
\hline 39 & Burkhardtsdorf, ab 1.8 .56 . . . & 2.50 & 2.50 \\
\hline 140 & Einsiedel bei Chemnitz, ab 1.8.56 . & 2.50 & 2.50 \\
\hline 141 & Lunzenau, ab $1.8 .56 \ldots . .$. & 1.75 & 1.75 \\
\hline 142 & Berggieshübel . . . . . . . . & 1.50 & 1.50 \\
\hline 143 & Brambach . . . . . . . & 2.- & 2.- \\
\hline 144 & $\begin{array}{l}\text { Elster, ab } 1852 \text { nur im Sommer, stän- } \\
\text { dig ab } 1855 \ldots \ldots\end{array}$ & 1.75 & 1.75 \\
\hline 145 & Gössnitz S. Á. (2 Typen) . . . . . . & 1.- & 1.- \\
\hline 146 & Großhartmannsdorf, ab 1.8 .50 . . & 1.50 & 1.50 \\
\hline 147 & Hartha bei Waldheim . . . . . . & 1.75 & 1.75 \\
\hline 148 & Hirschfelde . . . . . . & 1.- & 1.- \\
\hline 149 & Hohnstein bei Stolpen . . . . . . . & 2.- & 3.- \\
\hline 150 & Königswartha . . · · · · · · . & 1.50 & 1.50 \\
\hline 151 & 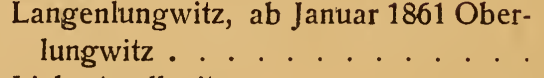 & 1.- & 1.- \\
\hline 152 & Liebertwolkwitz . . . . . . . & 1.75 & 1.75 \\
\hline 153 & Liebstadt . . . . . . . & 2.- & 2.- \\
\hline 154 & Markranstädt . . . & 1.50 & 1.50 \\
\hline 155 & Meuselwitz . . . . . & -.75 & -.75 \\
\hline 156 & Moritzburg . . . . . . . & 2.50 & 2.50 \\
\hline 157 & . . . . . . . . . & 2.- & 2.- \\
\hline
\end{tabular}




\begin{tabular}{|c|c|c|c|}
\hline 至 & Name des Postamtes & 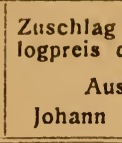 & $\begin{array}{l}\text { Kata } \\
\text { Market } \\
\text { be } \\
\text { Vappen }\end{array}$ \\
\hline & Oberkunnersdorf & 2.- & 2.- \\
\hline & & 1.- & 1.- \\
\hline & Oderwitz, ab 1.4.65 Nieder-Oderwitz & 1.50 & 1.50 \\
\hline & Ostritz $\ldots \ldots \ldots \ldots$ & 2.- & 2.- \\
\hline & Panschwitz . & 5.- & 5.- \\
\hline & Pausa & 2.- & 2.- \\
\hline & Reichenau bei Zittau, ab 1.2 .52 & 2.50 & 250 \\
\hline & he & -5 & -.50 \\
\hline & Sch & & 1.50 \\
\hline & Schle & & 1.50 \\
\hline & erg bei Brambach . & 3.- & 3- \\
\hline & Schö & 2.50 & 2.50 \\
\hline & rsdiorf & 1.- & 1.- \\
\hline & Siebenlehn . . . . . . . & 1.50 & 1.50 \\
\hline & $\begin{array}{l}\text { Silberstraße, am 1. Januar } 1859 \text { ver- } \\
\text { legt nach Wiesenburg. }\end{array}$ & 2.- & \\
\hline & 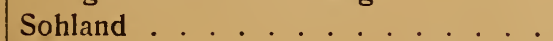 & 2.- & 2.- \\
\hline & & 1.50 & 1.50 \\
\hline & Weissenberg . . . . . . . . & 2.- & 2. - \\
\hline & $\begin{array}{l}\text { Wildenthal, Stempel am 1.4.62 über- } \\
\text { wiesen an Crottendorf. . . . . }\end{array}$ & 3.- & 3.- \\
\hline & Zwönitz . . . . . . . . & 1.5 & 1.50 \\
\hline & Oelsnitz b. Lichtenstein, ab 12.7.56 & 2.- & 2.- \\
\hline & Kieritzsch, ab $1.11 .56 \ldots \ldots \ldots$ & 2.50 & 10.- \\
\hline & $\begin{array}{l}\text { Neukirch am Hochwald, ab } 1.12 .56 \text {; } \\
\text { ab Februar } 1864 \text { Neukirch bei } \\
\text { Bischofswerda . . . . . . . }\end{array}$ & 3.- & \\
\hline & Rothenkirchen, ab 1.1.57. & 2.- & 2.- \\
\hline & & 2.- & 2.- \\
\hline & & & \\
\hline
\end{tabular}




\begin{tabular}{|c|c|c|c|}
\hline 造竞 & Name des Postamtes & \multicolumn{2}{|c|}{$\begin{array}{c}\text { Zuschlag zum Kata- } \\
\text { logpreis der Marken } \\
\text { Alisgabe } \\
\text { Johann Wappen }\end{array}$} \\
\hline 34 & löha, ab 1.6.57 . . . . . . . . & 2.50 & 2.50 \\
\hline 35 & Dresden-Görlitz, Zugpost, ab 1853 . & 1.50 & 3.- \\
\hline 86 & Pomsen, ab 1.7.57....... & 2.- & 1.50 \\
\hline 37 & Uhlstädt, ab 20.8.57. . . . . & 5.- & 5.- \\
\hline 88 & Mehltheuer bei Plauen, ab 1.9.57 & 5.- & 10.- \\
\hline 89 & Kreischa bei Dresden, ab 1.10.57. & 8.- & 8:- \\
\hline 190 & Laubegast, ab 1.10.57. . . . & 8.- & 8.- \\
\hline 191 & Lockwitz, ab 1.10.57. . & 5.- & 8.- \\
\hline 92 & Loschwitz, ab 1.11.57. & 3.- & 3.- \\
\hline 193 & Pillnitz, ab 1.11.57. . . . . . . & 2.- & 2.- \\
\hline 94 & Schönfeld bei Dresden, ab 1.11.57. & 5.- & 5.- \\
\hline 95 & Lohmen, ab 1.12.57. . . . . . & 10.- & 10.- \\
\hline 96 & Mylau, ab 1.12.57. & -.50 & -.50 \\
\hline 97 & $12.57 \ldots . .$. & 2.- & 2.- \\
\hline 98 & lorf bei Pulsnitz, ab 1.2.58 & 2.50 & 5.- \\
\hline 99 & , ab 1.2 .58 . . . . . . . & 5.- & 8. - \\
\hline 200 & en, ab 1.2.58. & $3-$ & 3.- \\
\hline 01 & Mutzschen, ab 1.3.58 . . . & 3.- & 3.- \\
\hline 02 & s, ab 1.4.58.... & 3.- & 3.- \\
\hline 03 & irchen bei Zschopau, ab 1.4.58 & 50 & 1.50 \\
\hline 04 & 1 , ab $1.5 .58 \ldots \ldots$ & 5.- & 5.- \\
\hline 05 & helshain, ab 1.6.58. & 10.- & 10.- \\
\hline 06 & sgrün, ab 1.7.58... & $3,-$ & 10.- \\
\hline 07 & $1, a b 1.10 .58$. . : & $5-$ & 5.- \\
\hline 08 & Klosterlausnitz, ab 15.10.58. & 5.- & 5.- \\
\hline 09 & Stauchitz, ab $1.12 .58 \ldots \ldots$ & 3.- & 3.- \\
\hline 10 & Buchholz bei Annaberg, ab 12.1.59. & -.50 & -.50 \\
\hline 211 & $\begin{array}{l}\text { Schmiedeberg bei Dippoldiswalde, } \\
\text { ab } 1.2 .59 \text {. . . . . . . . . }\end{array}$ & 4.- & 4.- \\
\hline . & Leipzig, Berliner Bahnhof, ab 1.2.59 & 50.- & 75.- \\
\hline
\end{tabular}




\begin{tabular}{|c|c|c|c|}
\hline \multirow{2}{*}{ 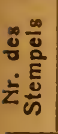 } & \multirow[t]{2}{*}{ Name des Postamtes } & \multicolumn{2}{|c|}{$\begin{array}{c}\text { Zuschlag zum Kata } \\
\text { logpreis der Marken } \\
\text { Ausgabe }\end{array}$} \\
\hline & & Johann & Wappen \\
\hline 213 & Siegmar, ab 15.3.59. & 1.- & 1.- \\
\hline 214 & Reitzenhain, $a b \quad 1.4 .59$ & 5.- & 5.- \\
\hline 215 & Possendorf, ab 1.5.59 & 5.- & 5.- \\
\hline 216 & Pommritz, ab 1.11.59. . . . & 5.- & 5. - \\
\hline 217 & Hainsberg-Deuben, ab 1.10.60: . & 1.- & 1.- \\
\hline 218 & $\begin{array}{c}\text { Lausa, ab } 1.11 .1860 \text {, ab } 1.8 .1866 \\
\text { Hermsdorf bei Dresden . . . . . }\end{array}$ & 10.- & 10.- \\
\hline 219 & Reuth bei Plauen, ab 1.8.61. . & 8.- & 8.- \\
\hline 220 & Cunewalde, ab 1.10.61. . . . . & 5.- & 5.- \\
\hline
\end{tabular}

Gitterstempel No. 205 war im Mai 1858 bei der Postexpedition Schwarzenberg-Bahnhof in Gebrauch.

Die Postanstalten ohne Eröffnungs-Datum bestanden bereits 1850 bei Einführung der Marken.

Nummernstempel auf der Ausgabe mit

Kopf des Königs Friedrich August

haben von Nr. 1-59 etwa den doppelten

" "60-133 , " dreifachen

" „134u.höher,, " fünffachen

Wert wie auf Marken der Ausgabe König Johann. 
Sächsische Postorte welche nur Ortsstempel ohne Nummernstempel benutzt haben.

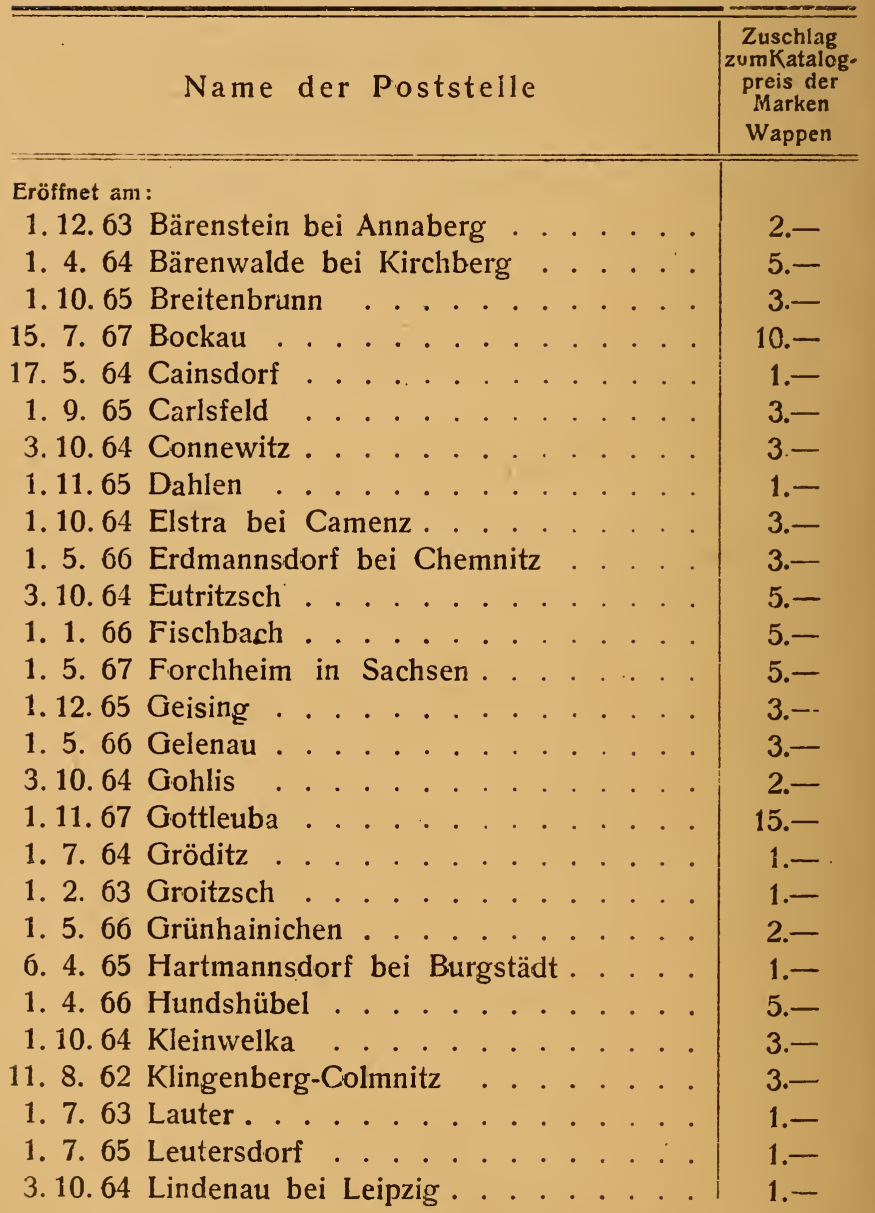




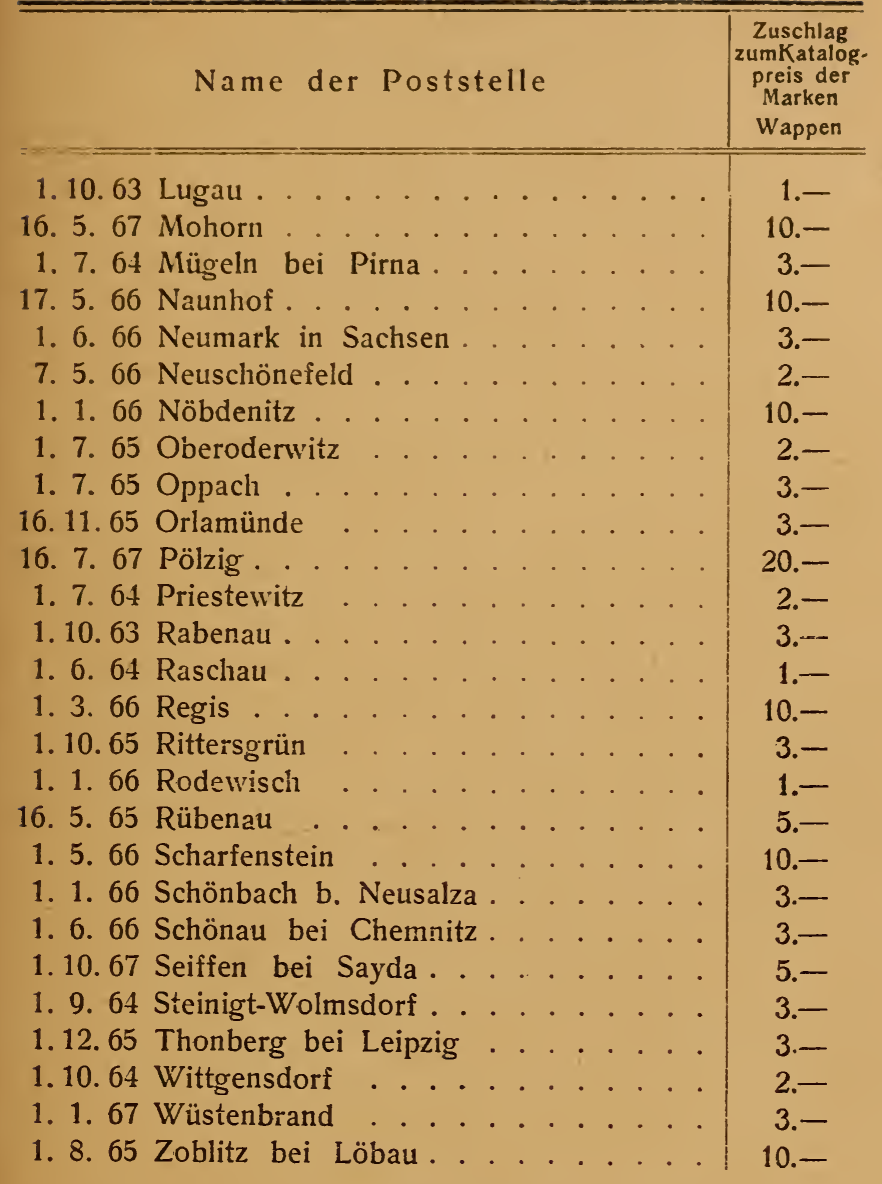

Auf Marken der Kopfausgabe König Johann kommen nur Klingenberg-Colmnitz (5.-) und Groitzsch (2.-) vor. 


\section{Name der Poststelle}

7. 8. 65 Dresden I (Jüdenhof)

1. 11.64 , II (Amalienstraße)

Zuschlag

zumKatalog.

preis der

Marken

Wappen

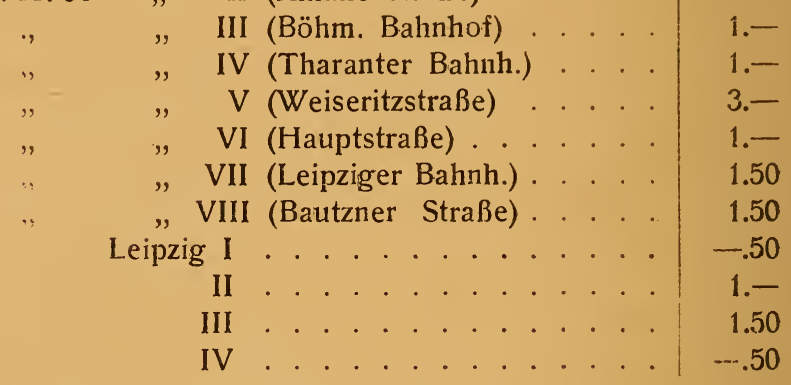

\section{Stadtpoststempel}

(meist rot oder violett):

Dresden I (Rundstempel

II-VIII (Langstempel . . . . . . . . . . . je 2.Leipzig-P(ost) - E(xpedition)

Nr. 1, 2, 3, 4 farbig. ......... . . je 2.-

Nr. 1 schwarz .. . . . . . . . . . . . -50

Stadtpost (ohne Stadtnamen):

schwarz farbig 


\section{Bahnhofsstempel.}

(Die Zahlen hinter den Namen bedeuten, daß diese Bahnhöfe auch Nuınmern-Stempel mit diesen Nummern führten, die fünf mit * verzeichneten Orte jedoch mit Ziffern in kleinerer Type.)

\begin{tabular}{|c|c|}
\hline Name des Postamtes & $\begin{array}{l}\text { Zuschlag } \\
\text { zumKatalog } \\
\text { preis der } \\
\text { Marken }\end{array}$ \\
\hline${ }^{*}$ Aue-Bahnhof (110) . . . . . & 1.- \\
\hline Annaberg-Bahnhof, ab 1.5.66 . . & 1.- \\
\hline Bautzen-Bahnhof . . . . . & 5.- \\
\hline Chemnitz-Bahnhof (8) . . . . . . . . . . & -.50 \\
\hline 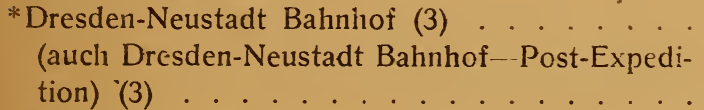 & $\begin{array}{l}-.50 \\
-.50\end{array}$ \\
\hline Dahlen-Bahnhof (93) . . & 2.- \\
\hline *Gössnitz-Bahnhof (145) . . . . . . . . . & $15 .-$ \\
\hline Leipzig-Bahnhof (4) . . . . . . . . . . . & -.50 \\
\hline Leipzig-Berliner Bahnhof (212) . . . . . . & 25.- \\
\hline Leipzig-Thüringer Bahnhof . . . . & 2.- \\
\hline Leipzig-Dresdner Bahnhof _. . . . . & -.50 \\
\hline Leipzig-Bahnliof Postexpedition (4) . . . & -.50 \\
\hline Pirna-Bahnhof . . . . . . . . . & -.50 \\
\hline Reichenbach-Bahnhof . . . . . . . & 2.- \\
\hline Riesa-Bahnhof-Postexpedition (165) . . & 2.- \\
\hline Schwarzenberg-Bahnhof (82) . . . . . & -.50 \\
\hline Wolkenstein-Bahnhof, ab 1.5.66 & 1.- \\
\hline *Zittau-Bahnhof (15) . . . . . & -.50 \\
\hline *Zwickau-Bahnhof (9) . . . . & 1.- \\
\hline Freiberg-Bahnhof . . . . . . . . . . & -.50 \\
\hline
\end{tabular}




\section{Postübernahmestempel.}

(Einzeiliger Typen- bzw. Langstempel, teilweise mit

Linieneinfassung.)

Altenburg

Bischofswerda

Meerane

Böhlen

Borna

Dresden

Dresden-Böhm. Bahnhof

Grossenhain

Gössnitz

Loebau

Lichtenstein

Leipzig

Meissen

Pirna

Reichenbach

Oschatz

Nicolai - Vorstadt Chemnitz

Ronneburg

Wilkau

Bahnhof Werdau

Wilsdruff

Diese Liste ist nicht als vollständig anzusehen; die einzelnen Stempel sind mit etwa $1-2 \mathrm{Mk}$. Aufgeld zu bewerten.

\section{Bahnpost(zug)stempel.}

(Die Zahlen in Klammern hinter den Namen der Bahnposten bedeuten, daß diese Bahnposten auch Nummerngitterstempel mit diesen Nummern in Gebrauch hatten.)

Annaberg-Chemnitz-Zug,

ab 1.5.66

Chemnitz-Riesa-Zug (7)

Dresden-Bodenbach-Zug

Dresden-Görlitz-Zug (185)

Dresden--Freiberg-Zug

Glauchau-Gera-Zug

Glauchau-Gössnitz-Zug
Leipzig-Dresden-Zug (6)

Leipzig-Hof-Zug (5)

Loebau-Zittau-Zug

Reichenbach-Eger-Zug ab 1.11.65

Riesa-Zwickau-Zug (7)

Zwickau-SchwarzenbergZug

Wert dieser verhältnismäßig häufigen Stempel je $50 \mathrm{Pf}$. bis $1 \mathrm{M}$. je nach Beschaffenheit. Etwas höher sind DresdenFreiberg und Reichenbach-Eger zu bewerten. 


\section{Briefsammlungen.}

$\begin{aligned} \text { Brief-Sammlung Nr. } 1 \text { (Gröditz) } & \text { abhängig von: } \\ \text { do. Nr. } 2 \text { (Schönfeld? Zeithain?) } & \text { Großenhain } \\ \text { do. Nr. } 3 \text { (Lausa b. Dresden) } & \\ \text { bis 31. 10.60 } & \text { Dresden } \\ \text { do. Nr. } 4 \text { (Neumark) bis 31.5.66 } & \begin{array}{l}\text { Fahrendem Postamt } \\ \text { Leipzig-Hof }\end{array} \\ \text { do. Nr. } 5 \text { (Ort bisher unbekannt) } & \end{aligned}$

Bärenstein bei Lauenstein, ab 1.10.63 Lauenstein

Bobenneukirchen

Carlsfeld, bis 31.8 .65

Crottendorf, bis 31.3 .62

Cunewalde, bis 30.9 .61

Dahlen, bis 31.10.65

Ehrenliain

Elstra, bis 30.9.64

Geising, bis 30.11 .65

Groitzsch, bis 31.1 .63

Gutenfürst, ab 1862

Hundshübel, ab 1.4.64 bis 31.3.66 Lindenau, bis 2.10.64

Nerchau

Lauter, bis 30.6.63

Mosel

Mutzschen, bis 28.2.58

Stadt-Neudorf, ab 1.9.64

Rabenau, bis 30.9.63

Oppach, ab 1.8.63 bis 31.3 .65

Remse

Reuth, bis 31.7.61

Schwepnitz, ab 1.5.64
Oelsnitz i. V.

Eibenstock

Scheibenberg

Bautzen

Luppa-Dahlen

Altenburg

Camenz

Altenberg (?)

Pegau

Fahrendem Postamt

Leipzig-Hof

Schneeberg-Neustädtel

Leipzig

Grimma

Aue

Zwickau

Wermsdorf

Dresden VII

Hainsberg

Neusalza

Waldenburg

Fahrendem Postamt

Leipzig-Hof

Königsbrück 
Uhlstädt, bis 19.8.57

Wittgensdorf, bis 30.9.64 abhängig von :

Kahla

Chemnitz

$\mathrm{Da}$ für diese Stempel nur ein ganz kleiner Kreis von Liebhabern in Frage kommt, dürfte eine Bewertung von 1-10 M. entsprechend sein.

Diese Stempel waren meist achteckige Langstempel mit Datum, aber ohne Stundenangabe, und mußten auf der Rückseite der Briefe angebracht werden, während die vorgesetzte Postanstalt die Marken auf der Vorderseite des Briefumschlages entwertete.

Zweifellos dürften noch andere Orte solche Briefsammlungen gehabt haben, konnten aber bisher noch nicht einwandfrei nachgewiesen werden.

Es wird gebeten, Nachrichten hierüber mit etwaigen Belegbriefen an Herrn Walter A. Opitz in Netzschkau i. Vogtl. gelangen zu lassen.

Feldpoststempel (s. Abbildung).

(Abdrucke meist farbig, seltener schwarz.) 1849.

F. Post-A. Sachsen . . . . . 25.1864.

F. Post-A. Sachsen . . . . . . 20.-

K. Sächs. Feldpost-Amt . . . . . 20.1866.

K. Sächs. Feldpost-Amt . . . . . . 5 5-

K. Sächs. Feld-Post-Expedition I . . . 10.do.

II . . . 15.- 


\section{Orts- und Schlagwort-Register.}

Die Ziffern in () bedeuten die Nummer des Gitterstempels.

\begin{tabular}{|c|c|c|c|}
\hline & Seite & & Seite \\
\hline Adorf (30) & 36 & Carlsfeld & 44,49 \\
\hline Altenberg (86) & 38 & Chemnitz (7), (8) 35, & 47,48 \\
\hline Altenburg (11) & 35,48 & Colditz (33) & 36 \\
\hline Annaberg (10) & $5,47,48$ & Connewitz & 44 \\
\hline Aue $(110)$ & 39,47 & Crimmitschau (34) & 36 \\
\hline Auerbach (31) & 36 & Crottendorf (176) & 41,49 \\
\hline Bärenstein & 44,49 & Cunewalde (220) & 43,49 \\
\hline Bärenwalde & 44 & Dahlen & 44,49 \\
\hline Bautzen (14) & 35,47 & Dahlen-Bahnhof (93) & 38,47 \\
\hline Berggießhübel (142) & 40 & Deutsch-Einsiedel (26) & 36 \\
\hline Bernstadt (111) & 39 & Dippoldiswalde $(63)$ & 37 \\
\hline Bischofswerda (48) & 37,48 & Döbeln (35) & 36 \\
\hline Bobenneukirchen & 49 & Dohna (39) & 36 \\
\hline Böhlen & 48 & Dreiermarke grün & 14 \\
\hline Borna (16) & 36,48 & do. rot & $7-13$ \\
\hline Brambach (143) & 40 & do. Fälschun & ngen 9 \\
\hline Brand (137) & 40 & Dresden $(1,3) \quad 35,46$ & 47,48 \\
\hline Brandis (138) & 40 & do. -Bodenbach (11 & 12) 39 \\
\hline Breitenbrunn & 44 & do. -Görlitz (185) & 42 \\
\hline Bockau & 44 & & \\
\hline Buchholz (210) & 42 & Ebersbach (113) & 39 \\
\hline Burgstädt (62) & 37 & Ehrenfriedersdorf (87) & 38 \\
\hline Burkhardtsdorf (139) & 40 & $\begin{array}{l}\text { Ehrenhain } \\
\text { Eibenstock (36) }\end{array}$ & $\begin{array}{l}49 \\
36\end{array}$ \\
\hline & 44 & Einsiedel (140) & 40 \\
\hline Camenz (32) & 36 & Eisenberg (64) & 37 \\
\hline
\end{tabular}


EIster (144)

Elsterberg (60)

Elstra

Elterlein (182)

Entwertungsstempel

Erdmannsdorf

Eutritzsch

Eybau (114)

Falkenstein (115)

Feldpost

Fischbach

Flöha (184)

Forchheim

Francokuvert-Ausschnitte

Frankenberg (37)

Frauenstein (116)

Freiberg (12)

Friedrich-August-Ausgabe

Frohburg (88)

Geising

Geithain (117)

Gelenau

Geringswalde (118)

Geyer (136)

Glashütte (40)

Glauchau (17)

Gohlis

Gößnitz (145)

Gottleuba

Grimma (44)

Gröditz
15-17

38

44,49

39

44

39

40

36

36,48

44 Kahla (67)

4.0, 47, 48 Kieritzsch (179)

44. Kirchbers (91)

37 Kleinwelka

44, 49 Klingenberg-Colmnitz

Hundshübel

Jägersgrün (206)

Jöhstadt (120)
Scite

44,49

36,48

(18)

(146) 40

42

38

39

38

44

49

42

37

43

36

40

37

44

42

37

40

37

40

Hummelshain (205) 42

44,49

42

Johann-Arsgabe 18-21

Johanngeorgenstacit (66) 37

39

37

41

38

44

44 


\begin{tabular}{|c|c|c|c|}
\hline \\
\hline \multicolumn{3}{|c|}{ Seite } & \multirow{2}{*}{$\begin{array}{r}\text { Seito } \\
40\end{array}$} \\
\hline Klingenthal (121) & 39 & Lunzenau (141) & \\
\hline Klosterlausnitz (208) & 42 & Luppa-Dahlen (93) & 38 \\
\hline Kohren (204) & 42 & & \\
\hline Königsbrück (68) & 37 & Marienberg (72) & 38 \\
\hline Königstein (69) & 37 & Markneukirchen (94) & 38 \\
\hline Königswartha (150) & 40 & Markranstädt (154) & 40 \\
\hline Kötschenbroda (25) & 36 & Meerane (53) & 37,48 \\
\hline Kreischa (189) & 42 & Mehltheuer (188) & 42 \\
\hline \multirow[t]{2}{*}{ Krögis (202) } & 42 & Meißen (19) & 36 \\
\hline & & Mcuselwitz (155) & 40 \\
\hline Langenlungwitz (151) & 40 & Mittweida (54) & 37 \\
\hline Laubegast $(190)$ & 42 & Mohorn & 45 \\
\hline Lauenstein (41) & 36 & Moritzburg (156) & 40 \\
\hline Lausa (218) & 43,49 & Mosel & 49 \\
\hline Lausigk (122) & 39 & Mügein (95) & 38,45 \\
\hline Lauter & 44,49 & Müinltroff (15i) & 40 \\
\hline \multirow{2}{*}{\multicolumn{2}{|c|}{ "-Berlin. Bahnhof $(212) 42$}} & Mülsen (42) & 36 \\
\hline & & Mutzschen (201) & 42,49 \\
\hline Leisnig (51) & 37 & Mylau (196) & 42 \\
\hline Lengefeld (123) & 39 & & \\
\hline Lengenfeld (70) & 37 & Naunhof & 45 \\
\hline Leutersdorf & 44 & Nerchau & 49 \\
\hline Lichtenstein (38) & 36,48 & Netzschkau (197) & 42 \\
\hline Liebertwolkwitz (152) & 40 & Neugersdorf (125) & 39 \\
\hline Liebstadt (154) & 40 & Neukirch (180) & 41 \\
\hline Limbach $(52)$ & 37 & Netukirchen (28) & 36 \\
\hline Lindenau & 44,48 & Neumark & 45,49 \\
\hline Löbau (45) & 37,48 & Neuschönefeld & 45 \\
\hline Lockwitz (191) & 42 & Neusalza (96) & 38 \\
\hline Lohmen (195) & 42 & Neustadt (73) & 38 \\
\hline Lommatzsch (92) & 38 & Nicolai-Vorstadt & 48 \\
\hline Loschwitz (192) & 42 & Nöbdenitz & 45 \\
\hline Lößnitz (71) & 37 & Nossen (74) & 38 \\
\hline Lucka (124) & 39 & & \\
\hline Lugan & 45 & Oberkunnersdorf (158) & 41 \\
\hline
\end{tabular}


Oberoderwitz

Oberwiesenthal (159)

Oderwitz (160)

Oederan (75)

Oelsnitz im Vogtl. (76) 38

Oelsnitz bei Lichtenstein

Olbernhau (97)

Oppach

Orlamünde

Oschatz (20)

Ostrau (135)

Ostritz (161)

Panschwitz (162)

Pausa (163)

Pegau (77)

Penig (55)

Pillnitz (193)

Pirna (21)

Plauen (46)

Pölzig

Pommritz (216)

Pomsen (186)

Possendorf (215)

Potschappel (78)

Preistafeln

Priestewitz

Pulsnitz (98)

Rabenau

Radeberg (79)

Radeburg (126)

Raschau

Regis
(178)

\section{8}

45,49

45

36,48

40

41

41

41

38

37

42

$36,47,48$

37

43

42

43

38

$35-50$

45

38

38

39

45

45
45

45,49
Reichenau (164).

Seite

41

Reichenbach (22) $36,47,48$

Reitzenhain (214) 42

Remse

49

Reuth (219) $\quad 43,49$

Riesa (80) ' 38,48

do. Bahnhof (165) 41,47

Rittersgrün

45

36

39

45

Rodewisch

Ronneburg (56)

Roßwein (57)

Rötha (99)

37, 48

37

38

41

45

38

38

45

39

39

41

41

42

39

35

45

45

Schönberg (168) 41

Schöneck (169) 41

Schönfeld (194) 42, 49

Schönhayda (Schönheide)

5 (103) 39

Schwarzenberg (82) 38,49 


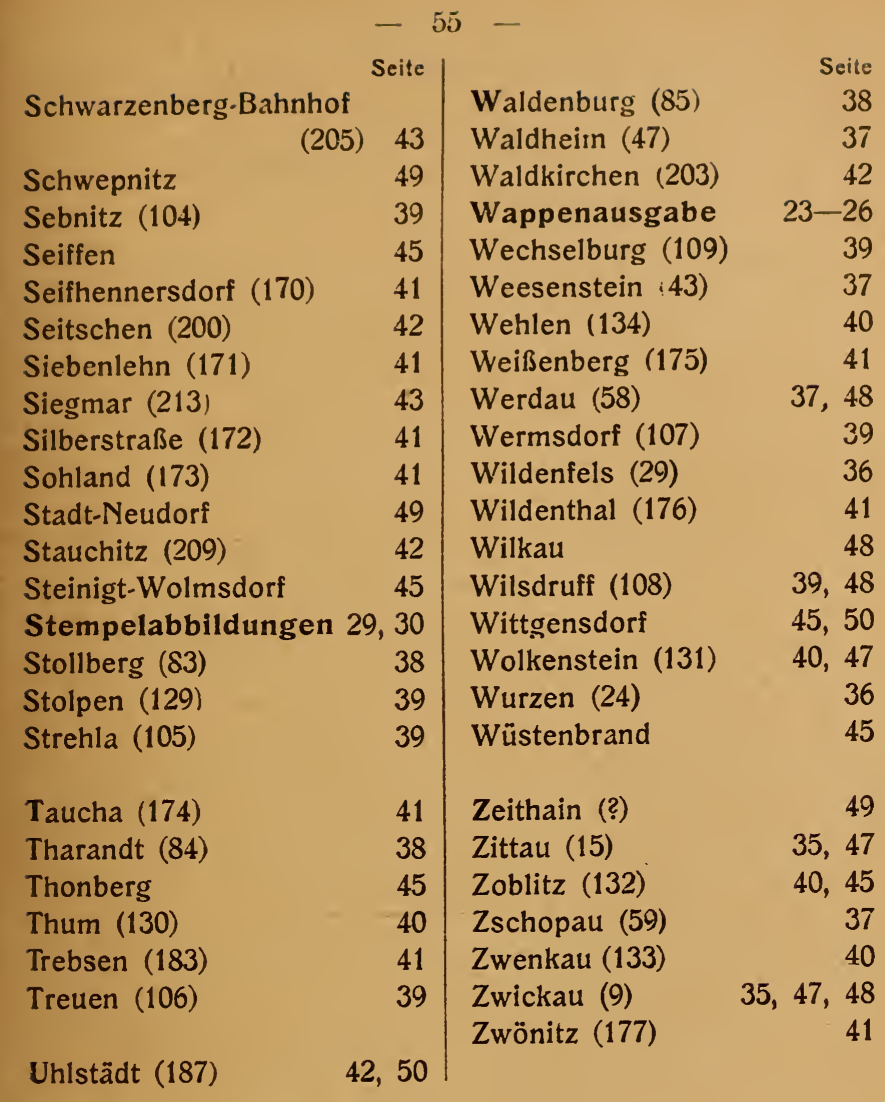




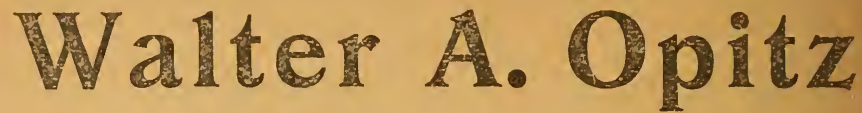

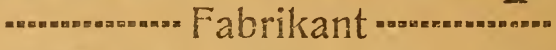 in Netzschkau i. Vogtl.}

sucht für seine Spezialsammlung von Sachsen: Ganzbriefe Friedrich August 1851, die Marken mit Ortsstempeln folgender Orte entwertet: Berggießhübel, Ebersbach, Falkenstein, Frauenstein, Frohburg, Geringswalde, Gößnitz, Großhartmannsdorf, Großschönau, Hartha, Lengefeld, Lengenfeld, Liebstadt, Markranstädt, Moritzburg, Mühltroff, Nossen, Ober-Cunnersdorf, Ob.-Wiesenthal, Ostritz, Pausa, Reichenau, Rötha, Sayda, Schellenberg, Schirgiswalde, Schönberg, Siebenlehn, Sohland, Stollberg, Stolpen, Taucha, Zöblitz, Zwönitz.

Friedrich August-Marken lose mit Nummerstempeln ab Nr. 60.

Ganzbriefe Johann mit Vollgitterstempel.

Wappenbriefe folgender Postorte: Bautzen-Bahnhof, Bockau, Crottendorf, Gottleuba, Pölzig, Lausa, Leipzig Berl. Bhf., Hummelshain.

Briefe aus Sachsen nach dem Ausland mit interessanten Frankaturen.

Frankierte såchsische Telegramm- und Postanweisungskuverts.

Quadratisch geschnittene Ausschnitte als Marken gebraucht nur auf ganzen Briefen.

Marken des Norddeutschen Postbezirks mit sächsischen Gitterstempeln.

Briefe mit rückseitigem Briefsammlungs. stempeln. 
$=1-x+x-x$ 


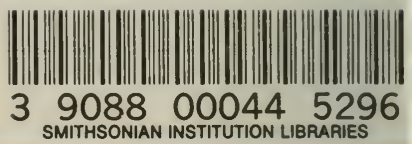

Druck von August Hoffmann

:: Leipzig

:: 